\title{
Awareness-dependent normalization framework of visual bottom-up attention
}

\author{
Shiyu Wang ${ }^{1,2}$, Ling Huang ${ }^{1,2}$, Qinglin Chen ${ }^{1,2}$, Jingyi Wang ${ }^{1,2}$, Siting Xu ${ }^{1,2}$, \\ Xilin Zhang $1,2,3,4 *$
}

${ }^{1}$ Key Laboratory of Brain, Cognition and Education Sciences, Ministry of Education, South China Normal University, Guangzhou, Guangdong 510631, China; ${ }^{2}$ School of Psychology, South China Normal University, Guangzhou, Guangdong 510631, China;

${ }^{3}$ Center for Studies of Psychological Application, South China Normal University, Guangzhou, Guangdong 510631, China; ${ }^{4}$ Guangdong Provincial Key Laboratory of Mental Health and Cognitive Science, South China Normal University, Guangzhou, Guangdong 510631, China

* Correspondence: xlzhang@m.scnu.edu.cn

Xilin Zhang, Ph.D. Professor

Key Laboratory of Brain, Cognition and Education Sciences, Ministry of Education,

School of Psychology, South China Normal University

Guangzhou, Guangdong, P.R. China, 510631

E-mail: xlzhang@m.scnu.edu.cn 


\section{Abstract}

Although bottom-up attention can improve visual performance with and without awareness, whether they are governed by a common neural computation remains unclear. Using a modified Posner paradigm with backward masking, we found that both the attention-triggered cueing effect with and without awareness displayed a monotonic gradient profile (Gaussian-like). The scope of this profile, however, was significantly wider with than without awareness. Subsequently, for each subject, the stimulus size was manipulated as their respective mean scopes with and without awareness while stimulus contrast was varied in a spatial cueing task. By measuring the gain pattern of contrast-response functions, we observed changes in the cueing effect consonant with changes in contrast gain for bottom-up attention with awareness and response gain for bottom-up attention without awareness. Our findings indicate an awareness-dependent normalization framework of visual bottom-up attention, placing a necessary constraint, namely, awareness, on our understanding of the neural computations underlying visual attention.

Keywords: visual bottom-up attention; normalization model; awareness; attention field; gradient profile 


\section{Introduction}

Covert attention, the selective processing of visual information at a given location in the absence of eye movements, can be attracted automatically by an exogenous cue, known as visual bottom-up attention (Corbetta and Shulman, 2002; Hegdé and Felleman, 2003; Kastner and Ungerleider, 2000; Koch and Ullman, 1985; Nakayama and Mackeben, 1989; Yantis and Jonides, 1984). Numerous studies have demonstrated that this bottom-up attention-triggered attraction can improve visual performance with (Beck and Kastner, 2005; Carrasco, 2011; Corbetta and Shulman, 2002; Kastner et al., 1997; Posner, 2016; Posner et al., 1980; Serences and Yantis, 2007) and without awareness in various paradigms, such as visual backward masking (Chen et al., 2016; Huang et al., 2020; Naccache et al., 2002; Zhang et al., 2012), crowding (Faivre and Kouider, 2011; Montaser-Kouhsari and Rajimehr, 2005), and continuous flash suppression (Bahrami et al., 2007; Hsieh et al., 2011; Jiang et al., 2006), as well as sub-threshold presentation (Bauer et al., 2009; Lin et al., 2009; Mulckhuyse and Theeuwes, 2010; Zhang and Fang, 2012) and the patient with blindsight (Kentridge et al., 1999a, 1999b, 2004). However, it's unclear whether there is a common neural computation governing bottom-up attention-triggered improvement in visual performance with and without awareness.

There has been a long-standing debate about the neural computations underlying visual bottom-up attention. Experiments examining how it modulates visual performance and neuronal activity in visual cortex have found disparate attentional effects on stimulus-evoked neural responses, such as the contrast-response function 
(CRF) that has often been used in the literature (Martinez-Trujillo and Treue, 2002;

Reynolds et al., 2000). Some have reported that attentional selection primarily enhances neural responses to high-contrast stimuli (response gain, Di Russo et al., 2001; Kim et al., 2007; Lee and Maunsell, 2009; Ling and Carrasco, 2006; McAdams and Maunsell, 1999; Morrone et al., 2002), whereas others have reported that attentional selection primarily enhances neural responses to medium-contrast stimuli (contrast gain, Li et al., 2008; Martínez-Trujillo and Treue, 2002; Reynolds and Chelazzi, 2004; Reynolds et al., 2000). Still others have reported that attentional selection either enhances the entire contrast range or produces a combination of both response-gain and contrast-gain changes (Buracas and Boynton, 2007; Huang and Dobkins, 2005; Murray, 2008; Pestilli et al., 2011; Williford and Maunsell, 2006).

Crucially, these ostensibly conflicting results of the gain changes induced by visual attention can be explained by the normalization model of attention (Boynton, 2009; Carandini and Heeger, 2012; Herrmann et al., 2010; Lee and Maunsell, 2009, 2010; Reynolds and Heeger, 2009; Reynolds et al., 1999), which proposes that attention-triggered improvements on perception hinge on two critical factors: the stimulus size and the attention field size. Changes in the relative size of these two factors can tip the balance between neuronal excitatory and inhibitory processes, thereby resulting in response-gain changes, contrast-gain changes, or various combinations of the two (Reynolds and Heeger, 2009). Specifically, this model predicts that attention increases contrast gain when the stimulus is small and the attention field is large and increases response gain when the stimulus is large and the 
attention field is small. Remarkably, previous psychophysical (Herrmann et al., 2010; Schallmo et al., 2020; Schwedhelm et al., 2016; Zhang et al., 2016), electroencephalography (Itthipuripat et al., 2014, 2019), and voxel-based functional magnetic resonance imaging (Hara et al., 2014) studies have reported that the patterns of behavioral performance, steady-state visual evoked potentials, and voxel-averaged neurometric functions, respectively, are all consistent with the predictions of normalization model of attention. However, little is known regarding whether visual bottom-up attention with and without awareness is governed by this common neural computation: normalization and how awareness could modulate the gain changes induced by attentional selection.

Here, using a modified Posner paradigm with backward masking, we first manipulated the distance between the cue and probe (Figure 1) to measure the attention filed of visual bottom-up attention with and without awareness (Distribution Experiments). We found that the Posner cueing effect with and without awareness were both a monotonic gradient profile with a center maximum falling off gradually in the surround (Gaussian-like, Figure 2). Thus, for each subject, we fit their gradient profiles with a Gaussian function and used the FWHM (full width at half maximum) bandwidth of the Gaussian to quantify their scopes. The scopes of gradient profiles, however, were significantly wider with than without awareness, which offers a unique opportunity to change the size of the attentional scope relative to the stimulus size. Thus, for each subject, the stimulus size was manipulated as their respective mean scopes of visual bottom-up attention with and without awareness while stimulus 
contrast was varied in a spatial cueing task (Normalization Experiments). We measured the gain pattern of CRFs on the spatial cueing effect derived by visible or invisible cues and empirically revealed an interaction between awareness and visual bottom-up attention: gain modulation depended on awareness, with a change in the spatial cueing effect consonant with a change in contrast gain for visible cues and response gain for invisible cues. Additionally, using the classical normalization model of attention (Reynolds and Heeger, 2009), we successfully simulated the scopes of visual bottom-up attention with and without awareness. Our results thus support important predictions of the normalization model of visual bottom-up attention and further reveal its dependence on awareness. 


\section{Results}

\section{Distribution Experiments}

The Distribution Experiment consisted of 3 experiments. In each visual field, the probe position was constant and the exogenous cue position varied in Experiment 1 (VCCP, Figure 1A), whereas Experiment 2 was a converse situation (CCVP, Figure 1D). In both Experiments 1 and 2, there were five possible distances between the exogenous cue and probe, ranging from D0 (cue and probe at the same location) through D4 (cue and probe four items away from each other). Subjects participated in Experiments 1 and 2 on two different days, and the order of the two experiments was counterbalanced across subjects. Experiment 3 checked the effectiveness of the awareness manipulation in both Experiments 1 and 2, and was always before them. Results showed that, our awareness manipulation was effective for both visible and invisible conditions (Figure S1). In both Experiments 1 (Figure 1C) and 2 (Figure 1F), each trial began with the fixation. A cue frame with (the cue condition) or without (the non-cue condition) exogenous cue was presented for 50-ms, followed by a $100-\mathrm{ms}$ mask (low- and high-contrast masks rendered the exogenous cue visible or invisible to subjects, respectively) and another 50-ms fixation interval. Then a probe line, orientating at $45^{\circ}$ or $135^{\circ}$ away from the vertical, was presented for 50 -ms. Subjects were asked to press one of two buttons as rapidly and correctly as possible to indicate the orientation of the probe $\left(45^{\circ}\right.$ or $\left.135^{\circ}\right)$. There was no significant difference in the false alarm rate, miss rate, or removal rate (i.e., correct reaction times shorter than 200 
$\mathrm{ms}$ and beyond three standard deviations from the mean reaction time in each condition were removed) across conditions (all $P>0.05$, Figure S2). The cueing effect for each distance (D0 to D4) was quantified as the difference between the reaction time of the probe task performance in the non-cue condition and that in the cue condition.

Figure $2 A$ shows the cueing effect of each condition for both Experiments 1 and 2; most of these cueing effects were significantly above zero, indicating that the bottom-up attention of the subject was attracted to the exogenous cue location, allowing them to perform more proficiently in the cue condition than the non-cue condition of the probe task. In both Experiments 1 and 2, a repeated measures ANOVA with awareness (visible and invisible) and distances (D0 to D4) as within-subjects factors showed that, the interaction between these two factors $\left(\right.$ Experiment 1: $\mathrm{F}_{4,60}=9.921, p<0.001, \eta_{\mathrm{p}}^{2}=0.398 ;$ Experiment 2: $\mathrm{F}_{4,60}=3.36, p=$ $0.015, \eta_{\mathrm{p}}^{2}=0.183$ ), the main effect of awareness (Experiment $1: \mathrm{F}_{1,15}=29.27, p<$ 0.001, $\eta_{\mathrm{p}}^{2}=0.661$; Experiment 2: $\mathrm{F}_{1,15}=72.26, p<0.001, \eta_{\mathrm{p}}^{2}=0.828$ ), and the main effect of distances (Experiment 1: $\mathrm{F}_{4,60}=80.08, p<0.001, \eta_{\mathrm{p}}^{2}=0.842$; Experiment 2: $\left.\mathrm{F}_{4,60}=86.30, p<0.001, \eta_{\mathrm{p}}^{2}=0.852\right)$ were all significant. Subsequent post hoc paired $t$ tests revealed that the cueing effect decreased gradually with the distance in both Experiments 1 (the visible condition, D0 versus D1: $\mathrm{t}_{15}=6.56, p<0.001, \eta_{\mathrm{p}}^{2}=3.388$, D1 versus D2: $\mathrm{t}_{15}=3.68, p=0.023, \eta_{\mathrm{p}}^{2}=1.900, \mathrm{D} 2$ versus D3: $\mathrm{t}_{15}=4.36, p=0.006, \eta_{\mathrm{p}}^{2}$ $=2.251, \mathrm{D} 3$ versus D4: $\mathrm{t}_{15}=3.18, p=0.063, \eta_{\mathrm{p}}^{2}=1.642$; the invisible condition, D0 versus D1: $\mathrm{t}_{15}=6.44, p<0.001, \eta_{\mathrm{p}}^{2}=3.326$, D1 versus $\mathrm{D} 2: \mathrm{t}_{15}=4.13, p=0.009, \eta_{\mathrm{p}}^{2}=$ 
2.133, D2 versus D3: $\mathrm{t}_{15}=1.60, p=1.000, \eta_{\mathrm{p}}^{2}=0.826$, D3 versus D4: $\mathrm{t}_{15}=0.60, p=$ $1.000, \eta_{\mathrm{p}}^{2}=0.310$ ) and 2 (the visible condition, D0 versus D1: $\mathrm{t}_{15}=6.72, p<0.001, \eta_{\mathrm{p}}^{2}$ $=3.470, \mathrm{D} 1$ versus D2: $\mathrm{t}_{15}=5.70, p<0.001, \eta_{\mathrm{p}}^{2}=2.943, \mathrm{D} 2$ versus D3: $\mathrm{t}_{15}=1.28, p=$ $1, \eta_{\mathrm{p}}^{2}=0.661$, D3 versus D4: $\mathrm{t}_{15}=2.08, p=0.554, \eta_{\mathrm{p}}^{2}=1.074$; the invisible condition, D0 versus D1: $\mathrm{t}_{15}=9.12, p<0.001, \eta_{\mathrm{p}}^{2}=4.710, \mathrm{D} 1$ versus D2: $\mathrm{t}_{15}=2.27, p=0.001, \eta_{\mathrm{p}}^{2}$ $=1.172, \mathrm{D} 2$ versus D3: $\mathrm{t}_{15}=0.50, p=1.000, \eta_{\mathrm{p}}^{2}=0.258, \mathrm{D} 3$ versus D4: $\mathrm{t}_{15}=0.40, p=$ $\left.1.000, \eta_{\mathrm{p}}^{2}=2.207\right)$. These results indicated that bottom-up attention-triggered cueing effects with and without awareness were both a monotonic gradient profile with a center maximum falling off gradually in the surround.

Subsequently, to further assess the shape of bottom-up attentional modulation, we fitted a monotonic model and two non-monotonic models to the average cueing effect across distances (D0 to D4) in both visible and invisible conditions. The monotonic model was implemented as the Gaussian function, and the two non-monotonic models were implemented as the Mexican Hat (i.e., a negative second derivative of a Gaussian function) and Polynomial functions (Fang and Liu, 2019; Fang et al., 2019; Finke et al., 2008). To compare these three models to our data, we first computed the Akaike information criterion (AIC, Akaike, 1973) and Bayesian information criterion (BIC, Schwarz, 1978) with the assumption of a normal error distribution. Then, we calculated the likelihood ratio (LR) and Bayes factor (BF) of the monotonic model (Gaussian) over non-monotonic models (Mexican Hat and Polynomial) based on AIC (Burnham and Anderson, 2002) and BIC (Wagenmakers, 2007) approximation, respectively. Results showed that, in both Experiments 1 and 2, 
the LR/BF (Table 1, top) strongly favored the Gaussian model over both the Mexican Hat and Polynomial models (Figure 2A). Notably, we also conducted similar model comparisons for each subject' data and found that the Gaussian model was favored over both the Mexican Hat and Polynomial models in 11 and 10 for Experiment 1, in 9 and 9 for Experiment 2, out of 16 subjects, during the visible and invisible conditions, respectively (Figure S4). In addition, we pooled the data from Experiments 1 and 2 together and further provided the same qualitative conclusion. The LR/BF (Table 1, top) strongly favored the Gaussian model over both the Mexican Hat and Polynomial models (Figure 2A, bottom). The model comparison based on fitting individual data also demonstrated that the Gaussian model was favored over both the Mexican Hat and Polynomial models in 12 and 10 out of 16 subjects during the visible and invisible conditions, respectively (Figure S4). These results further constituted strong evidence for the monotonic gradient profile of visual bottom-up attention with and without awareness.

\begin{tabular}{|c|c|c|c|c|c|c|c|c|c|c|c|c|}
\hline & \multicolumn{4}{|c|}{ Experiment $1(n=16)$} & \multicolumn{4}{|c|}{ Experiment $2(n=16)$} & \multicolumn{4}{|c|}{ Experiments 1\&2 $(n=16)$} \\
\hline & \multicolumn{2}{|c|}{ visible } & \multicolumn{2}{|c|}{ invisible } & \multicolumn{2}{|c|}{ visible } & \multicolumn{2}{|c|}{ invisible } & \multicolumn{2}{|c|}{ visible } & \multicolumn{2}{|c|}{ invisible } \\
\hline & M & $\mathrm{P}$ & M & $\mathrm{P}$ & M & $\mathrm{P}$ & M & $\mathrm{P}$ & M & $\mathrm{P}$ & M & $\mathrm{P}$ \\
\hline \multirow[t]{4}{*}{ G } & 3.82 & 20.00 & 46.65 & $9.88 * 10^{3}$ & 30.07 & $5.06 * 10^{2}$ & $5.03 * 10^{5}$ & $9.53 * 10^{6}$ & 10.30 & $1.01 * 10^{2}$ & $2.51 * 10^{4}$ & $6.33 * 10^{5}$ \\
\hline & \multicolumn{4}{|c|}{ Experiment $1(n=14)$} & \multicolumn{4}{|c|}{ Experiment $2(n=14)$} & \multicolumn{4}{|c|}{ Experiments 1\&2 $(n=14)$} \\
\hline & \multicolumn{2}{|c|}{ visible } & \multicolumn{2}{|c|}{ invisible } & \multicolumn{2}{|c|}{ visible } & \multicolumn{2}{|c|}{ invisible } & \multicolumn{2}{|c|}{ visible } & \multicolumn{2}{|c|}{ invisible } \\
\hline & M & $\mathrm{P}$ & M & $\mathrm{P}$ & M & $\mathrm{P}$ & M & $\mathrm{P}$ & M & $\mathrm{P}$ & M & $\mathrm{P}$ \\
\hline G & 14.32 & $1.89 * 10^{2}$ & $1.12 * 10^{3}$ & $3.86 * 10^{3}$ & 4.26 & 27.87 & $3.03 * 10^{3}$ & $1.85 * 10^{4}$ & 22.35 & $4.44 * 10^{2}$ & $2.49 * 10^{3}$ & $1.19 * 10^{4}$ \\
\hline
\end{tabular}

Our results indicated that the spatial focus of visual bottom-up attention with and without awareness were best explained by the monotonic (Gaussian) rather than the 
non-monotonic models (Mexican Hat and Polynomial). To quantitatively examine the scope of bottom-up attentional modulation, we fitted the cueing effects from D0 to D4 with a Gaussian function and used the FWHM bandwidth of the Gaussian to quantify their scopes. Results showed that the fitted FWHM bandwidth was significantly larger in the visible than the invisible condition for both Experiments $1\left(\mathrm{t}_{15}=3.015, p=\right.$ $0.009, \eta_{\mathrm{p}}^{2}=1.557$, Figure $2 B$, top $)$ and $2\left(\mathrm{t}_{15}=4.863, p<0.001, \eta_{\mathrm{p}}^{2}=2.511\right.$, Figure $2 B$, middle), as well as for the pooled data from two experiments $\left(\mathrm{t}_{15}=4.745, p<0.001, \eta\right.$ ${ }_{\mathrm{p}}^{2}=2.450$, Figure $2 \mathrm{~B}$, bottom), indicating a wider scope of bottom-up attention with than without awareness. Notably, this awareness-dependent scope of bottom-up attention here could be explained by the difference in cueing effect between the visible and inviable conditions. To examine this issue, we calculated the correlation coefficients between our fitted FWHM bandwidths and cueing effects across individual subjects. If a wider scope of visual bottom-up attention with than without awareness is derived by a greater cueing effect in the visible than the invisible condition, then we would observe a significant correlation between these two measures across individual subjects. However, for Experiments 1 and 2, as well as for the pooled data from the two, compared to the invisible condition, the increased FWHM bandwidth was not significantly correlated with the increased peak cueing effect (i.e., the cueing effect of D0, Experiment 1: $r=-0.294, p=0.269, \eta_{\mathrm{p}}^{2}=0.086$; Experiment 2: $\mathrm{r}=0.428, p=0.098, \eta_{\mathrm{p}}^{2}=0.183$; Experiments $1 \& 2: \mathrm{r}=0.0484, p=$ $0.859, \eta_{\mathrm{p}}^{2}=0.002$ ) or the mean of cueing effects across distances (Experiment 1: $\mathrm{r}=$ -0.156, $p=0.564, \eta_{\mathrm{p}}^{2}=0.024$; Experiment 2: $\mathrm{r}=0.343, p=0.194, \eta_{\mathrm{p}}^{2}=0.118$; 
Experiments $1 \& 2: \mathrm{r}=0.0619, p=0.820, \eta_{\mathrm{p}}^{2}=0.004$ ) in the visible condition (Figure 2C), which against the cueing effect explanation.

More importantly, to directly exclude this explanation, we examined the scope of bottom-up attention during visible and invisible conditions with no significant difference in the cueing effect between the two conditions. We manipulated the cueing effect of visible condition by decreasing the luminance of its cue (which was still visible to subjects, Figure S1). Fourteen of our 16 subjects repeated Distribution Experiments using these low luminance cues and the same repeated measures ANOVA indicated that our manipulation was effective by showing that, in both Experiments 1 and 2, the main effect of awareness (Experiment 1: $\mathrm{F}_{1,13}=3.283, p=$ 0.093, $\eta_{\mathrm{p}}^{2}=0.202$; Experiment 2: $\mathrm{F}_{1,13}=1.472, p=0.247, \eta_{\mathrm{p}}^{2}=0.102$ ) was not significant (Figure 2D). Our model comparisons also provided the same qualitative conclusion that the Gaussian model was strongly favored over both the Mexican Hat and Polynomial models with the LR/BF (Table 1, bottom), based on both the group (Figure 2D) and individual (Figure S4) data. More importantly, we confirmed that the fitted FWHM bandwidth was significantly larger in the visible than the invisible condition for both Experiments $1\left(\mathrm{t}_{13}=3.732, p=0.003, \eta_{\mathrm{p}}^{2}=2.070\right.$, Figure $2 E$, top $)$ and $2\left(\mathrm{t}_{13}=2.561, p=0.024, \eta_{\mathrm{p}}^{2}=1.421\right.$, Figure $2 \mathrm{E}$, middle $)$, as well as for the pooled data from the two $\left(\mathrm{t}_{13}=3.752, p=0.002, \eta_{\mathrm{p}}^{2}=2.081\right.$, Figure $2 E$, bottom $)$. Additionally, these increased FWHM bandwidths in the visible condition relative to the invisible condition weren't significantly predicted by the increased peak cueing effect (Experiment 1: $\mathrm{r}=0.227, p=0.434, \eta_{\mathrm{p}}^{2}=0.052$; Experiment $2: \mathrm{r}=0.094, p=0.749, \eta_{\mathrm{p}}^{2}$ 
$=0.009 ;$ Experiments $\left.1 \& 2: \mathrm{r}=0.151, p=0.607, \eta_{\mathrm{p}}^{2}=0.023\right)$ or the mean of cueing effects across distances (Experiment 1: $\mathrm{r}=0.334, p=0.243, \eta_{\mathrm{p}}^{2}=0.112$; Experiment 2 : $\mathrm{r}=0.140, p=0.634, \eta_{\mathrm{p}}^{2}=0.020$; Experiments $\left.1 \& 2: \mathrm{r}=0.235, p=0.419, \eta_{\mathrm{p}}^{2}=0.055\right)$ (Figure 2F). Thus, our findings indicate a gradient profile of visual bottom-up attention with and without awareness, and show a wider scope of visual bottom-up attention with than without awareness.

\section{Normalization Experiments}

Our Distribution Experiments demonstrated an awareness-dependent scope of visual bottom-up attention, which offers a unique opportunity to change the size of the attention field relative to the stimulus, differentially modulating the gain of bottom-up attentional selection. Thus, for each subject, the diameter of grating (Figure 3A) was manipulated as their respective mean FWHM bandwidth of the Gaussian with and without awareness, i.e., the diameter of grating $=\left(\mathrm{FWHM}_{\mathrm{V}}+\mathrm{FWHM}_{\mathrm{I}}\right) / 2$, where FWHM $_{V}$ and FWHM $_{I}$ are the fitted FWHM bandwidth of the Gaussian model for the visible and invisible conditions in Distribution Experiments, respectively. Under this configuration, the attentional field was larger and smaller than the stimulus size for the visible and invisible cues, yielding a pattern that qualitatively resembled contrast gain or response gain, respectively (Figure $3 B$ ). To examine these predictions, we used a modified version of the Posner paradigm to measure the cueing effect induced by the visible or invisible cue, as shown in Figure $3 C$. In both two conditions, an exogenous cue, a low-luminance ring, randomly appeared at the center of 9 positions in left or right hemifield with equal probability, followed by a 100-ms mask (low- and 
high-contrast for visible and invisible conditions, respectively) and another 50-ms fixation interval. Then, a pair of gratings was presented for $33 \mathrm{~ms}$ in the left and right hemifields and subjects were asked to press one of two buttons to indicate the orientation of one of two gratings; each was presented at five different contrasts $(0.02$, $0.08,0.15,0.40$, and 0.70 , the contrasts of both gratings were identical on any given trial and covaried across trials in random order). A response cue at gratings offset indicated the target grating, yielding congruent cue (the exogenous cue matched the response cue, half the trials) and incongruent cue (mismatched, half the trials) conditions (Figure 3C). Comparing performance accuracy $\left(d^{\prime}\right)$ for congruent and incongruent trials revealed the spatial cueing effect for each target contrast.

The mean $d^{\prime}$ plotted as psychometric functions of stimulus contrast and awareness (visible and invisible) are shown in Figure 4A: the visible condition yielded a pattern that qualitatively resembled contrast gain, and the invisible condition yielded a pattern that qualitatively resembled response gain (see also Figure S5A). The measured psychometric function for awareness (visible and invisible) and trial conditions (congruent and incongruent) was fit with the standard Naka-Rushton equation (Naka and Rushton, 1966). The two parameters $c_{50}$ (the contrast yielding half-maximum performance) and $d^{\prime}{ }_{\max }$ (asymptotic performance at high-contrast levels) determined contrast gain and response gain, respectively. The exponent $n$ (slope) was fixed at 2 in the current analysis (Carandini and Heeger, 2012; Herrmann et al., 2010; Reynolds and Heeger, 2009; Zhang et al., 2016). The $d^{\prime}{ }_{\max }$ for awareness (visible and invisible) and trial conditions (congruent and incongruent) are shown in 
Figure 4 and were submitted to a repeated-measures ANOVA with awareness and trial condition as within-subjects factors. The main effect of awareness $\left(\mathrm{F}_{1,12}=8.915, p=\right.$ $\left.0.011, \eta_{\mathrm{p}}^{2}=0.426\right)$, the main effect of the trial condition $\left(\mathrm{F}_{1,12}=70.366, p<0.001, \eta_{\mathrm{p}}^{2}=\right.$ $0.854)$ and the interaction between these two factors $\left(\mathrm{F}_{1,12}=71.311, p<0.001, \eta_{\mathrm{p}}^{2}=\right.$ 0.856) were all significant. Post hoc paired $t$ tests showed that $d^{\prime}{ }_{\max }$ of congruent trials was higher than that of incongruent trials for the invisible condition $\left(t_{12}=\right.$ 12.166, $p<0.001, \eta_{\mathrm{p}}^{2}=7.024$, Figure $4 C$, left $)$, but not for the visible condition $\left(\mathrm{t}_{12}=\right.$ $1.784, p=1.000, \eta_{\mathrm{p}}^{2}=1.030$, Figure $4 B$, left $) ; d^{\prime} \max$ for the invisible condition was higher than that for the visible condition in the congruent trials $\left(\mathrm{t}_{12}=5.163, p<0.001\right.$, $\left.\eta_{\mathrm{p}}^{2}=2.981\right)$, but not in the incongruent trials $\left(\mathrm{t}_{12}=1.098, p=0.294, \eta_{\mathrm{p}}^{2}=0.634\right)$. Similarly, for the $c_{50}$, the main effect of awareness $\left(\mathrm{F}_{1,12}=5.468, p=0.037, \eta_{\mathrm{p}}^{2}=\right.$ $0.313)$, the main effect of trial condition $\left(\mathrm{F}_{1,12}=45.342, p<0.001, \eta_{\mathrm{p}}^{2}=0.791\right)$ and the interaction between these two factors $\left(F_{1,12}=52.415, p<0.001, \eta_{\mathrm{p}}^{2}=0.814\right)$ were all significant. Post hoc paired $t$ tests showed that $c_{50}$ of congruent trials was lower than that of incongruent trials for the visible condition $\left(\mathrm{t}_{12}=-9.303, p<0.001, \eta_{\mathrm{p}}^{2}=-5.371\right.$, Figure 4D, left), but not for the invisible condition $\left(\mathrm{t}_{12}=-0.577, p=0.575, \eta_{\mathrm{p}}^{2}=-0.333\right.$, Figure $4 E$, left); $c_{50}$ for the visible condition was lower than that for the invisible condition in the congruent trials $\left(\mathrm{t}_{12}=-5.074, p<0.001, \eta_{\mathrm{p}}^{2}=-2.929\right)$, but not in the incongruent trials $\left(\mathrm{t}_{12}=0.023, p=0.982, \eta_{\mathrm{p}}^{2}=0.013\right)$. These results thus suggest that gain modulation of bottom-up attentional selection depends on awareness.

To evaluate further the role of awareness in the gain modulation of visual bottom-up attention, we calculated the correlation coefficients between the relative 
size of the attention field to the stimulus [i.e., $\left(\mathrm{FWHM}_{\mathrm{V}}-\mathrm{FWHM}_{\mathrm{I}}\right) / 2$, where FWHM $_{V}$ and FWHM are the fitted FWHM bandwidth of the Gaussian model for the visible and invisible conditions, respectively] and psychophysical measures ( $d^{\prime}{ }_{\max }$ and $\left.c_{50}\right)$ across individual subjects. The relative size of the attention field to the stimulus in the visible condition significantly correlated with the $c_{50}$ difference between congruent and incongruent trials $\left(\mathrm{r}=-0.602, p=0.029, \eta_{\mathrm{p}}^{2}=0.362\right.$, Figure $4 D$, right $)$, but not with the $d^{\prime}{ }_{\max }$ difference between congruent and incongruent trials $(\mathrm{r}=-0.110$, $p=0.721, \eta_{\mathrm{p}}^{2}=0.012$, Figure $4 B$, right $)$. Conversely, the relative size of the attention field to the stimulus in the invisible condition significantly correlated with the $d^{\prime}{ }_{\max }$ difference between congruent and incongruent trials $\left(\mathrm{r}=0.591, p=0.033, \eta_{\mathrm{p}}^{2}=0.349\right.$, Figure $4 C$, right), but not with the $c_{50}$ difference between congruent and incongruent trials $\left(\mathrm{r}=-0.011, p=0.973, \eta_{\mathrm{p}}^{2}=0.0001\right.$, Figure $4 E$, right $)$. These results thus demonstrate a close relationship between awareness and gain modulation of visual bottom-up attentional selection (response gain and contrast gain changes in psychophysical performance).

In addition, to further confirm this awareness-dependent normalization framework of visual bottom-up attention, we simulated our empirical data with the normalization model of attention (Figure 5A and Figure S5B) using custom Matlab scripts based on the code of Reynolds and Heeger (2009) with 4 free parameters: the gain of attention $[A(x, \theta)]$, separately optimized for visible and invisible conditions, the normalization constant $\sigma$, and a scaling parameter to linearly scale simulated values to performance $\left(d^{\prime}\right)$. Given the simulated attention fields $[A(x, \theta)]$ are in arbitrary units; 
only the relative values are meaningful (Reynolds and Heeger, 2009), in both the visible and invisible conditions, we thus calculated the correlation coefficients between the simulated and experimental attention fields (i.e., the FWHM) across individual subjects. In both two conditions, the simulated attention fields (marginally) significantly correlated with the experimental attention fields (the visible conditions: $r$ $=0.792, p=0.001, \eta_{\mathrm{p}}^{2}=0.627$; the invisible conditions: $\mathrm{r}=0.505, p=0.079, \eta_{\mathrm{p}}^{2}=$ 0.255 , Figure $5 B$ ), further conforming that manipulating subjects' awareness could modulate the field of visual bottom-up attention, which, in turn, affected its normalization processes. 


\section{Discussion}

We examined the underlying neural computations of visual bottom-up attention with and without awareness and found the following results. First, we found support for previous neurophysiological (Connor et al., 1996, 1997; Schall and Hanes, 1993), psychophysical (Downing 1988; Handy et al., 1996; Henderson and Macquistan, 1993; LaBerge, 1983; Mangun and Hillyard, 1988; Posner, 2016; Robertson et al., 2013; Shulman et al. 1985), eye movement (Tkacz-Domb and Yeshurun, 2018), electroencephalographic (Couperus and Lydic, 2019; Eimer, 1997; Mangun and Hillyard, 1988), and functional magnetic resonance imaging (Brefczynski-Lewis et al., 2009) studies, indicating that the bottom-up attention-triggered improvements in visual performance with and without awareness were both a monotonic gradient profile with a center maximum falling off gradually in the surround (Gaussian-like). Second, however, the scope of this gradient profile was significantly wider with than without awareness, which offers a unique opportunity to change the size of the bottom-up attentional scope relative to the stimulus size. Thus, for each subject, the stimulus size was manipulated as their respective mean scopes of bottom-up attention with and without awareness while stimulus contrast was varied. By measuring the gain pattern of CRFs on the spatial cueing effect derived from visible or invisible exogenous cues, we observed a change in the spatial cueing effect consistent with a change in contrast gain for visible cues and in response gain for invisible cues. Finally, using the classical normalization model of attention (Reynolds and Heeger, 2009), we successfully simulated the scopes of visual bottom-up attention with and without 
awareness, indicating an awareness-dependent normalization framework of visual bottom-up attention. In addition, our results cannot be explained by the eye movement since subjects' eye movements were small and their eye position distributions were statistically indistinguishable for visible and invisible conditions (Figure S3).

\section{Gradient profile of visual bottom-up attention with and without awareness}

Compared to a monotonic gradient profile evident in previous and our studies, a number of neurophysiological (Moran and Desimone, 1985; Schall and Hanes, 1993; Schall et al., 2004), psychophysical (Bahcall and Kowler, 1999; Mounts, 2000; Müller et al., 2005), and brain imaging (Boehler et al., 2009, 2011; Hopf et al., 2006, 2010; Müller and Kleinschmidt, 2004) studies, as well as a computational model (Tsotsos et al., 1995, 2008) have reported a center-surround (i.e., the Mexican Hat) profile where a zone of sensory attenuation surrounds a center region of facilitation. We suggested that this striking discrepancy in the literature findings might be due to two different factors. One is the experimental task or paradigm. Specifically, for example, in Hopf et al. (2006) where inhibition was seen surrounding the locus of spatial attention, subjects were asked to search for a target (i.e., the exogenous cue in our study), which appeared randomly to change the spatial focus of attention and thus was task-relevant. While they measured the event-related magnetic field response elicited by a task-irrelevant probe that appeared or was absent with equal probability. In our study, by contrast, we used a modified Posner paradigm to measure the probe's attentional effect induced by the exogenous cue. Subjects were asked to discriminate 
the orientation $\left(45^{\circ}\right.$ or $\left.135^{\circ}\right)$ of the probe (Figure 1$)$, thus the exogenous cue was task-irrelevant while the probe was task-relevant. Obviously, the probe was the distractor (task-irrelevant) and the target (task-relevant) in Hopf et al.'s and our studies, respectively. Directing attention to the target could conflate perceptual and post-perceptual mechanisms of attention, thus eliminating or strongly attenuating the suppression effect.

The other that may influence the spatial profile of visual bottom-up attention is whether the exogenous cue was presented with (e.g., Hopf et al., 2006) or without (e.g., the current study) the distractors. As is known to all, the biased competition model of attention (Desimone and Duncan, 1995) proposes that attention operates when multiple stimuli compete for access to neural representation, and this competition occurs when multiple stimuli fall within a neuron's receptive field. In this case, distractors within a receptive field are suppressed while attended stimuli are enhanced. Additionally, Tsotsos and colleagues $(1995,2008)$ proposed the selective tuning model, which directly suggested that the inhibitory zone surrounding the attended item results from top-down propagation of a winner-take-all mechanism that attenuates irrelevant upstream connections iteratively from one hierarchical level down to the next. Thus, without the distractor, these irrelevant upstream connections across the visual cortical processing hierarchy could be eliminated or strongly attenuated, which, in turn, affects the inhibitory zone surrounding the attended target, in other words, resulting in the gradient rather than center-surround profile of attentional modulation. 


\section{A wider scope of visual bottom-up attention with than without awareness}

Our study provided convening evidence for a wider scope of visual bottom-up attention with than without awareness. Although this result cannot be explained by the difference in the cueing effect between visible and invisible conditions (Figure 2D), it could be argued that this result can be derived by that the visible relative to invisible condition has involved some degree of endogenous attention as well. Particularly, for Experiment 1 (VCCP, Figure 1A), compared to the invisible condition, subjects during the visible condition knew on each trial that the exogenous cue appeared randomly across the possible 9 positions and could have therefore directed endogenous attention to all, which might increase the attentional set (Couperus and Lydic, 2019; Gibson and Kelsey, 1998), thus yielding a wider scope of attentional modulation. Critically, it is important to note that in our study, the task required subjects to discriminate the orientation $\left(45^{\circ}\right.$ or $\left.135^{\circ}\right)$ of the probe; the exogenous cue was never task-relevant. Thus, subjects did not need to direct endogenous attention to these task-irrelevant cues. More importantly, this endogenous attention (i.e., the attentional set) explanation couldn't account for the same qualitative conclusion in our Experiment 2 (CCVP, Figure 1D) since the same exogenous cue was always presented at the center of 9 locations during both the visible and invisible conditions. In other words, there was the same attentional set between two conditions. If the wider scope of visual bottom-up attention with than without awareness is derived by this potential endogenous attention (i.e., the attentional set driven by the observer's 
knowledge of the cue location), then we should have observed a similar scope of the two. However, our data show that this is not the case.

Our findings can be viewed as identifying an awareness-dependent scope of visual bottom-up attention. Note that this conclusion is based on a report-based paradigm in which subjects overtly push a button to report their percept. Several studies have argued that such report-based paradigms could be modulated by factors that are not directly related to the scope of attentional modulation, such as higher-level strategies, response history, experience, learning, response biases, and personality (Yeshurun, 2019). In addition, using a no-report paradigm, such as recording subjects' pupillary light responses, Tkacz-Domb and Yeshurun (2018) revealed that the scope of attentional modulation was twofold larger than that estimated using the traditional report-based paradigm. It is important to note that subjects in our study performed exactly the same task between visible and invisible conditions (Figure $1 C$ and $1 F$ ), thus the awareness-dependent scope of visual bottom-up attention evident here cannot be explained by this discrepancy between the report-based and no-report paradigms. However, the underlying neural basis of awareness-dependent scope of visual bottom-up attention could depend on whether subjects overtly report their percept.

On the one hand, several theories of conscious awareness, including the neuronal global workspace (Dehaene and Changeux, 2011; Mashour et al., 2020), information integration (Koch et al., 2016; Tononi et al., 2016), and higher-order (Lau and Rosenthal, 2011) theories propose that the neural activity in frontoparietal cortex is 
essential for conscious awareness. Similar to our study, evidence from those theories typically used the report-based paradigm in which subjects overtly report their percept, and showed that a broad frontoparietal network of areas could be activated during various tasks that contrast perceived stimuli with invisible stimuli (Dehaene and Changeux, 2011; Mashour et al., 2020). Thus, although speculative, it is plausible that the wider scope of bottom-up attention with than without awareness evident in our study may result from the increased activity in frontoparietal cortical areas. On the other hand, several studies have argued that such report-based paradigms do not dissociate the brain regions required for pure conscious experience from those involved in conscious access and reportability (Koch et al., 2016; Tsuchiya et al., 2015). Those studies, by contrast, found that posterior rather than frontoparietal cortical areas were activated when using a no-report paradigm, such as recording eye movements and pupil dilation (Aru et al., 2012; Frässle et al., 2014). In other words, the awareness-dependent scope of visual bottom-up attention is more likely to be mediated by posterior cortical areas when using no-report paradigm in which there is not any overt report. Consequently, further work is needed using both report-based and no-report paradigms to examine the difference in scope of visual bottom-up attention with and without awareness, as well as their distinct neural mechanisms.

\section{Awareness-dependent normalization framework of visual bottom-up attention}

The most parsimonious account of our results is that visual bottom-up attention interacts with the normalization processes depending on awareness. Importantly, this 
result cannot be explained by a number of factors, such as the strength of cueing effect, post-stimulus cue, or an involvement of endogenous attention. First, both the visible and invisible cues were the same with those in Distribution Experiments and no significant difference in cueing effect was found between the two (Figure 2D). Second, although previous studies have suggested that the post-stimulus cue (for example, the response cue in our study) can influence not only subjects' non-perceptual decision (Eckstein et al., 2013) but also the perception of stimuli presented before it (Pestilli et al., 2011; Sergent et al., 2013), the response cue in our study was totally randomized and uninformative about the target grating; we thus believe that our psychophysical results cannot be explained by the response cue. Finally, subjects knew before each trial that the discrimination task was to be performed on one of two gratings and could have therefore directed endogenous attention to both, and thus it is not known exactly how exogenous attention and this endogenous attention combine (Herrmann et al., 2010). However, subjects in our study performed exactly the same task between visible and invisible conditions, this potential combination thus couldn't account for the observed awareness-dependent normalization processes of visual bottom-up attention.

Our data can be interpreted by a hypothesis that behavioral performance is limited by the neuronal activity with an additive, independent, and identically distributed noise, and the decision-making process with a maximum-likelihood decision rule (Jazayeri and Movshon, 2006; Pestilli et al., 2009). Performance accuracy $d^{\prime}$, used in both previous (Herrmann et al., 2010; Zhang et al., 2016) and our 
studies here, is proportional to the signal-to-noise ratio of the underlying neuronal responses. Thus, it can parallel reflect any change in neuronal CRFs in our study. Indeed, we found that a change in the cueing effect consonant with a change in contrast gain of CRF for bottom-up attention with awareness and a change in response gain of CRF for bottom-up attention without awareness (Figure 4A). These awareness-dependent gain modulations of visual bottom-up attentional selection support and extend the normalization model of attention (Boynton, 2009; Carandini and Heeger, 2012; Herrmann et al., 2010; Lee and Maunsell, 2009, 2010; Reynolds and Heeger, 2009; Reynolds et al., 1999). This model proposes that, in the absence of attention (e.g., in the incongruent cue condition), two factors determine the firing rate of a visually responsive neuron. One is the stimulus drive (excitatory component) determined by the contrast of the stimulus placed in the receptive field of a neuron. The other is the suppressive drive (inhibitory component) determined by the summed activity of other neighboring neurons, which serves to normalize the overall spike rate of the given neuron via mutual inhibition (Heeger, 1992; Reynolds and Heeger, 2009). Attention (e.g., in the congruent cue condition) modulates the pattern of neural activity by altering the balance between these excitatory and inhibitory components, depending on the relative sizes of the attention field to the stimulus size, and thereby exhibiting response gain changes, contrast gain changes, and various combinations of the two. In our study, given the scope of visual bottom-up attention was significantly wider with than without awareness (Figure 2), for each subject, the size of the target stimuli in the spatial cueing task was manipulated as their respective mean scopes of 
visual bottom-up attention with and without awareness (Figure $3 A$ ). Thus relative to the stimulus size, the broadened attention field by visible exogenous cues led to contrast gain changes because attentional gain was applied equally to the stimulus and suppressive drives. Conversely, the narrowed attention field by invisible exogenous cues led to response gain changes because attentional gain enhanced the entire stimulus drive, but only enhanced the center of the suppressive drive. Indeed, using the classical normalization model of attention, we successfully simulated these broadened and narrowed attention fields of visible and invisible cues, respectively (Figure 5), further supporting an awareness-dependent normalization framework of visual bottom-up attention.

Notably, evidence from neurophysiological and brain imaging studies indicate controversies concerning the brain regions involved in visual bottom-up attention, such as subcortical structures (Fecteau and Munoz, 2006; Shipp, 2004), visual (Mazer and Gallant, 2003; Zhang et al., 2012) and frontoparietal (Bisley and Goldberg, 2010; Corbetta and Shulman, 2002; Moore and Zirnsak, 2017; Squire et al., 2013) cortical areas. An important factor of this controversy is the awareness, which determines whether the realized neural substrate reflects the pure bottom-up attention or not (Chen et al., 2016; Huang et al., 2020; Zhang et al., 2012). Intriguingly, our results are consistent with this idea by showing an awareness-dependent normalization framework of visual bottom-up attention. Although normalization as a neural computation likely occurs throughout the whole brain (Carandini and Heeger, 2012; Schmitz and Duncan, 2018), the observed neural correlates of its interaction with 
visual bottom-up attention could also depend on awareness, and further studies will shed light on this issue using neurophysiological or brain imaging techniques.

\section{Conclusions}

In sum, we conclude that manipulating subjects' awareness can modulate the scope of visual bottom-up attentional modulation, which, in turn, affects its normalization processes. Our study provides, to the best of our knowledge, the first experimental evidence supporting an awareness-dependent normalization framework of visual bottom-up attention, thereby furthering our understanding of the neural computations underlying visual attention, the relationship between attention and awareness, and how they interactively shape our experience of the world. 


\section{Methods and Materials}

\section{Subjects}

A total of 16 human subjects (8 male, 19-26 years old) were involved in the study. All of them participated in Distribution Experiments, fourteen of them repeated the Distribution Experiments with decreased luminance of visible cues, and the following Normalization Experiments. They were naïve to the purpose of the study. They were right-handed, reported normal or corrected-to-normal vision, and had no known neurological or visual disorders. They gave written, informed consent, and our procedures and protocols were approved by the human subjects review committee of School of Psychology at South China Normal University.

\section{Apparatus}

Visual stimuli were displayed on an IIYAMA color graphic monitor (model: HM204DT; refresh rate: $60 \mathrm{~Hz}$; resolution: $1,280 \times 1,024$; size: 22 inches) at a viewing distance of $57 \mathrm{~cm}$. Subjects' head position was stabilized using a chin rest. A white fixation cross was always present at the center of the monitor.

\section{Distribution Experiments}

\section{Stimuli}

As illustrated in Figure 1, each texture stimulus contained 18 positions (the possible locations of the exogenous cue and probe) settled at an iso-eccentric distance from fixation $\left(8.27^{\circ}\right.$ of visual angle); a half of them were located in the left visual field and 
the other half was located in the right visual field. The center-to-center distance between two neighboring positions was $1.35^{\circ}$. The exogenous cue was a low-luminance ring $\left(8.9 \mathrm{~cd} / \mathrm{m}^{2}\right.$; inner dimeter: $0.909^{\circ}$; outer dimeter: $\left.0.961^{\circ}\right)$ while the probe was a rectangle of $0.104^{\circ} \times 0.831^{\circ}$ in visual angle and was orientated at $45^{\circ}$ or $135^{\circ}$ away from the vertical. Low- and high-contrast masks, which had the same grid as the texture stimulus, rendered the exogenous cue visible or invisible (confirmed by a two-alternative forced choice, 2AFC) to subjects, respectively. Each mask ring contained two pairs of orthogonal circular arcs, one pair was white (19.3 and $79.8 \mathrm{~cd} / \mathrm{m}^{2}$ for Low- and high-contrast masks, respectively) and the other pair was black (11.3 and $0.01 \mathrm{~cd} / \mathrm{m}^{2}$ for Low- and high-contrast masks, respectively). The ring in the mask had the same size as the exogenous cue in the texture stimulus (Figure $1 B$ and $1 E)$.

\section{Procedure}

The Distribution Experiment consisted of 3 experiments. In each visual field, the probe position was constant and the exogenous cue position varied in Experiment 1 (i.e., the varied cue with constant probe, VCCP, Figure 1A), whereas Experiment 2 was a converse situation (i.e., the constant cue with varied probe, CCVP, Figure 1D). In both Experiments 1 and 2, there were five possible distances between the exogenous cue and probe, ranging from D0 (cue and probe at the same location) through D4 (cue and probe four items away from each other). Subjects participated in Experiments 1 and 2 on two different days, and the order of the two experiments was counterbalanced across subjects. Experiment 3 checked the effectiveness of the 
awareness manipulation in both Experiments 1 and 2, and was always before them. In both Experiments 1 and 2, each trial began with the fixation. A cue frame with (the cue condition) or without (the non-cue condition) exogenous cue was presented for 50-ms, followed by a 100-ms mask (low- and high-contrast in visible and invisible conditions, respectively, confirmed by Experiment 3, Figure S1) and another 50-ms fixation interval. Then a probe line, orientating at $45^{\circ}$ or $135^{\circ}$ away from the vertical, was presented for 50-ms. Subjects were asked to press one of two buttons as rapidly and correctly as possible to indicate the orientation of the probe $\left(45^{\circ}\right.$ or $\left.135^{\circ}\right)$. The cueing effect for each distance (D0 to D4) was quantified as the difference between the reaction time of the probe task performance in the non-cue condition and that in the cue condition.

Differently, Experiment 1 consisted of 16 blocks of 96 trials, 48 for the left visual field and 48 for the right visual field. In each block and each visual field, an exogenous cue was equiprobably and randomly presented at one of the 9 positions in 40 trials (the cue condition) and was absent in the remaining 8 trials (the non-cue condition). The probe was always presented at the center of 9 positions (i.e., the VCCP, Figure 1A). Experiment 2 consisted of 16 blocks of 80 trials, 40 for the left visual field and 40 for the right visual field. In each block and each visual field, an exogenous cue always appeared (the cue condition) or was absent (the non-cue condition) in the center of 9 positions with equal probability; the probe appeared equiprobably and randomly across the possible 9 positions (i.e., the CCVP, Figure 1D). 
The stimuli and procedure in the 2AFC experiment (i.e., Experiment 3) were the same as those in Experiments 1 and 2, except that no probe was presented (Figure S1). Experiment 3 checked the effectiveness of the awareness manipulation in Experiments 1 and 2, and was always before them. In Experiment 3, all subjects underwent a $2 \mathrm{AFC}$ task to determine whether the masked cue was visible or invisible in a criterion-free way. After the presentation of a masked cue frame, subjects were asked to indicate which side (upper left or upper right) from the fixation they thought the cue appeared. Their performances were significantly higher or not statistically different from chance for all possible distances (D0 to D4), providing an objective confirmation that the cue was indeed visible or invisible to subjects, respectively.

\section{Model fitting and comparison}

For each subject and each condition (visible and invisible), we fitted a monotonic model and two non-monotonic models to the averaged cueing effect. The monotonic model was implemented as the Gaussian function, and the two non-monotonic models were implemented as the Mexican Hat (i.e., a negative second derivative of a Gaussian function) and Polynomial functions (Fang and Liu, 2019; Fang et al., 2019;

Finke et al., 2008):

$$
\begin{aligned}
& \text { Gaussian function: } y=y 0+\frac{2 A}{w \sqrt{2 \pi}} e^{-2\left(\frac{x}{w}\right)^{2}} \\
& \text { Mexican Hat function: } y=\frac{2 H}{\sqrt{3 m} \pi^{\frac{1}{4}}} e^{-\frac{x^{2}}{2 m^{2}}}\left(1-\frac{x^{2}}{m^{2}}\right)+y 1 \\
& \text { Polynomial function: } y=a x^{4}+b x^{2}+c
\end{aligned}
$$

where $y$ is the cueing effect, $x$ is the distance between the cue and probe (i.e., D0 to 
D4); $w, A$, and $y 0$ are the three parameters controlling the shape of the Gaussian function; $m, H$, and $y l$ are three parameters controlling the shape of the Mexican Hat function; $a, b$, and $c$ are the three free parameters controlling the shape of the Polynomial function (note that we used a fourth-order polynomial without the odd-power terms for the cueing effect since the symmetric shape). To compare these three models to our data, we first computed the Akaike information criterion (AIC, Akaike, 1973) and Bayesian information criterion (BIC, Schwarz, 1978), with the assumption of a normal error distribution:

$$
\begin{aligned}
& A I C=N \ln \left(\frac{R S S}{N}\right)+2 K+\frac{2 K(K+1)}{N-K-1} \\
& B I C=N \ln \left(\frac{R S S}{N}\right)+K \ln (N)
\end{aligned}
$$

where $N$ is the number of observations, $K$ is the number of free parameters, and $R S S$ is residual sum of squares (Raftery, 1999). Then, we further calculated the Likelihood ratio (LR) and Bayes factor (BF) of the monotonic model (Gaussian) over non-monotonic models (Mexican Hat and Polynomial) based on AIC (Burnham and Anderson, 2002) and BIC (Wagenmakers, 2007) approximation, respectively:

$$
\begin{aligned}
& L R=e^{\left(\frac{A I C_{N}-A I C_{M}}{2}\right)} \\
& B F=e^{\left(\frac{B I C_{N}-B I C_{M}}{2}\right)}
\end{aligned}
$$

where $A I C_{M}$ and $B I C_{M}$ are for the monotonic (Gaussian) model, $A I C_{N}$ and $B I C_{N}$ are for non-monotonic (Mexican Hat and Polynomial) models. The results indicated that, during each condition (visible and invisible), the monotonic (Gaussian) model was strongly favored over the non-monotonic models (Mexican Hat and Polynomial) 
(Figure 2). Thus, to quantitatively examine the scope of attentional modulation, we fit the averaged cueing effects from D0 to D4 with a Gaussian function and used the FWHM (full width at half maximum) bandwidth of the Gaussian to quantify their scopes:

$$
F W H M=w \sqrt{\ln (4)}
$$

where $w$ is the fitted width of the Gaussian function.

\section{Normalization Experiments}

\section{Stimuli}

As illustrated in Figure 3C, the exogenous cue of Normalization Experiments was the same as those in the Distribution Experiment 2, i.e., the exogenous cue always appeared in the center of 9 positions in left or right hemifield at $8.27^{\circ}$ eccentricity. The probe was a pair of gratings (spatial frequency: 1.7 cycles $/^{\circ}$; phase: random) that were presented at the exogenous cue's locations in the left and right hemifields. The gratings were presented at five possible contrasts: 0.02, 0.08, 0.15, 0.40, and 0.70. For each subject, the diameter of grating was manipulated as their respective mean FWHM bandwidth for the Gaussian of bottom-up attention with and without awareness in Distribution Experiments (Figure 3A):

$$
D=\frac{F W H M_{V}+F W H M_{I}}{2}
$$

where $\boldsymbol{D}$ is the diameter of grating, $F W H M_{V}$ and $F W H M_{I}$ are the fitted FWHM bandwidth of the Gaussian model in the visible and invisible conditions during Distribution Experiments (Experiments 1 \& 2), respectively. 


\section{Procedure}

The Normalization Experiment consisted of 2 experiments. Each trial began with central fixation. The exogenous cue, a low-luminance ring, randomly appeared at the center of 9 positions in left or right hemifield with equal probability, followed by a 100-ms mask (low- and high-contrast for visible and invisible conditions, respectively) and another 50-ms fixation interval. Then, a pair of gratings (with identical contrasts) was presented for $33 \mathrm{~ms}$ in the left and right hemifields, one of which was the target. Subjects were asked to press one of two buttons to indicate the orientation of the target grating (leftward or rightward tilted) and received auditory feedback if their response was incorrect. The target grating was indicated by a peripheral $100 \mathrm{~ms}$ response cue $\left(0.4^{\circ}\right.$ black circular arc) above one of the grating locations, but not at the grating location to avoid masking. A congruent cue was defined as a match between the exogenous cue location and response cue location (half the trials); an incongruent cue was defined as a mismatch (half the trials) (Figure 3C). Subjects were explicitly told that the exogenous cue was randomized and uninformative about the target location. The Normalization Experiment consisted of two sessions (visible and invisible), with the two sessions occurring on different days; the order of the two sessions was counterbalanced across subjects. Each session consisted of 64 blocks; each block had 80 trials, from randomly interleaving 16 trials from each of the five contrasts. Contrast varied from trial to trial in randomly shuffled order, and stimuli were presented briefly (i.e., $33 \mathrm{~ms}$ ) to avoid any possible dependence of attentional state on stimulus contrast. The attentional effect for each grating contrast was 
quantified as the difference between the performance accuracy $\left(d^{\prime}\right)$ in the congruent and incongruent cue conditions.

\section{Psychophysical data analysis}

To quantitatively examine the pattern of gain (either contrast or response gain) separately for bottom-up attention with and without awareness, for each subject, performance-i.e., $d^{\prime}=\mathrm{z}$ (hit rate) - z (false alarm rate)—was assessed across experimental blocks for each contrast and each trial condition (congruent and incongruent). A rightward response to a rightward stimulus tilt was (arbitrarily) considered to be a hit, and a rightward response to a leftward stimulus was considered to be a false alarm. For each subject, the mean $d^{\prime}$ CRFs obtained for congruent and incongruent trials were fit with the standard Naka-Rushton equation (Naka and Rushton, 1966):

$$
d^{\prime}(c)=d^{\prime}{ }_{\max } \frac{c^{n}}{c^{n}+c_{50}^{n}}
$$

where $d^{\prime}$ is performance as a function of contrast (c), $d^{\prime}{ }_{\text {max }}$ determines the asymptotic performance at high contrasts, $c_{50}$ is the contrast corresponding to half the asymptotic performance, and $n$ is an exponent that determines the slope of the CRFs. The two parameters $d^{\prime}{ }_{\max }$ and $c_{50}$ determined response gain and contrast gain, respectively. We estimated these two parameters for each condition while $n$ (slope) was fixed at 2 according to previous studies (Carandini and Heeger, 2012; Herrmann et al., 2010; Reynolds and Heeger, 2009; Zhang et al., 2016). 


\section{Model simulations}

The normalization model of attention (Reynolds and Heeger, 2009) computes the response of an arbitrary single neuron to a given set of stimuli as:

$$
\begin{aligned}
& R(x, \theta ; c)=\frac{A(x, \theta) E(x, \theta ; c)}{S(x, \theta ; c)+\sigma} \\
& S(x, \theta ; c)=s(x, \theta) *[A(x, \theta) E(x, \theta ; c)]
\end{aligned}
$$

where $R(x, \theta ; c)$ is the response of a neuron with its receptive field centered at $x$ and its orientation tuning centered at $\theta$, receiving stimulus input with contrast $c, A(x, \theta)$ is the attention field, $E(x, \theta ; c)$ is the stimulus drive of the population of neurons evoked by contrast $c, \sigma$ is the normalization constant, $S(x, \theta ; c)$ is the effect of the normalizing pool and represents the excitatory drive convolved by the suppressive surround, $s(x, \theta)$ is the suppressive field, and $*$ is convolution. Applying the attention field in the model can yield either a change in response gain, a change in contrast gain, or a combination of the two, depending on the stimulus size and the extent of the attention field relative to the sizes of the stimulation and suppressive fields. Relative to the stimulus size, the broadened attention field led to contrast gain changes since attentional gain $(\lambda)$ was applied equally to the stimulus ( $\alpha c, \alpha$ is the constant gain of the neuron receiving it's preferred input with contrast $c$ ) and suppressive drives $(S)$. The responses of a model neuron can be approximated as:

$$
\begin{aligned}
& R(x, \theta ; c)=\frac{\lambda E(x, \theta ; c)}{s(x, \theta) *[\lambda E(x, \theta ; c)]+\sigma} \\
& R(c) \approx \frac{\lambda \alpha c}{\lambda S+\sigma}=\frac{\alpha c}{S+\frac{\sigma}{\lambda}}
\end{aligned}
$$

Conversely, the narrowed attention field led to response gain changes since attentional 
gain $(\lambda)$ enhanced the entire stimulus drive $(\alpha c)$, but its impact on the denominator $S$ $+\sigma$ is much minimal. In this case:

$$
R(c) \approx \frac{\lambda \alpha c}{S+\sigma}
$$

Our results supported these predictions of the normalization model of attention by showing that manipulating subjects' awareness could modulate the field of visual bottom-up attention, which, in turn, affected its normalization processes (see Figure 4). To further confirm this awareness-dependent normalization framework of visual bottom-up attention, we simulated our empirical data using custom Matlab scripts based on the code of Reynolds and Heeger (2009) with 4 free parameters: the gain of attention $[A(x, \theta)]$, separately optimized for visible and invisible cue conditions, the normalization constant $\sigma$, and a scaling parameter to linearly scale simulated values to $d^{\prime}$. Given the simulated attention fields $[A(x, \theta)]$ are in arbitrary units; only the relative values are meaningful (Reynolds and Heeger, 2009), in both the visible and invisible conditions, we thus calculated the correlation coefficients between the simulated attention field and experimental attention fields (i.e., the FWHM) across individual subjects (see Figure 5). 


\section{Acknowledgements}

We thank David Heeger and Ruyuan Zhang for valuable comments. This work was supported by the National Outstanding Youth Science Fund Project of National Natural Science Foundation of China (Project: 32022032), the National Natural Science Foundation of China (General Program: 31871135), and the Key Realm R\&D Program of Guangzhou (202007030005).

\section{Additional information}

\section{Funding}

Funder Grant reference number Author

National Outstanding

Youth Science Fund

Project of National Natural Project 32022032

Xilin Zhang

Science Foundation of

China

National Natural Science

Foundation of China

Project 31871135

Xilin Zhang

(General Program)

Key Realm R\&D Program

Project 202007030005

Xilin Zhang

of Guangzhou

The funders had no role in study design, data collection and interpretation, or the decision to submit the work for publication. 


\section{Author contributions}

Shiyu Wang, Conceptualization, Formal analysis, Investigation, Methodology, Writing

- original draft, Writing - review and editing; Ling Huang, Qinglin Chen, Jingyi Wang,

Siting $\mathrm{Xu}$, Formal analysis, Investigation, Methodology; Xilin Zhang,

Conceptualization, Formal analysis, Supervision, Funding acquisition, Investigation,

Methodology, Writing - original draft, Writing - review and editing

\section{Author ORCIDs}

Xilin Zhang: https://orcid.org/0000-0003-0449-934X

\section{Ethics}

Human subjects: They study was approved by the human subjects review committee of School of Psychology at South China Normal University.

\section{Additional files}

\section{Data availability}

The data and custom-built MATLAB scripts of this study are available at Open

Science Framework: https://osf.io/gqzvm/. 


\section{References}

Akaike H. 1973. Information theory as an extension of the maximum likelihood principle. In: Petrov, B.N., and Csaki, F. (Eds). Second International Symposium on Information Teory. (Budapest: Akademiai Kiado).

Aru J, Bachmann T, Singer W, Melloni L. 2012. Distilling the neural correlates of consciousness. Neurosci Biobehav Rev. 36: 737-746.

Bahcall DO, Kowler E. 1999. Attentional interference at small spatial separations. Vision Res. 39: $71-86$.

Bahrami B, Lavie N, Rees G. 2007. Attentional load modulates responses of human primary visual cortex to invisible stimuli. Curr Biol. 17: 509-513.

Bauer F, Cheadle SW, Parton A, Müller HJ, Usher M. 2009. Gamma flicker triggers attentional selection without awareness. Proc Natl Acad Sci USA 106: 1666-1671.

Beck DM, Kastner S. 2005. Stimulus context modulates competition in human extrastriate cortex. Nat Neurosci. 8: 1110-1116.

Bisley JW, Goldberg ME. 2010. Attention, intention, and priority in the parietal lobe. Annu Rev Neurosci. 33: $1-21$.

Boehler CN, Tsotsos JK, Schoenfeld MA, Heinze HJ, Hopf JM. 2009. The center-surround profile of the focus of attention arises from recurrent processing in visual cortex. Cereb Cortex 19: 982-991.

Boehler CN, Tsotsos JK, Schoenfeld MA, Heinze HJ, Hopf JM. 2011. Neural mechanisms of surround attenuation and distractor competition in visual search. J Neurosci. 31: 5213-5224.

Boynton GM. 2009. A framework for describing the effects of attention on visual responses. Vision Res. 49: 1129-1143.

Brefczynski-Lewis JA, Datta R, Lewis JW, DeYoe EA. 2009. The topography of visuospatial attention as revealed by a novel visual field mapping technique. J Cogn Neurosci. 21: $1447-1460$.

Buracas GT, Boynton GM. 2007. The effect of spatial attention on contrast response functions in human visual cortex. J Neurosci. 27: 93-97.

Burnham KP, Anderson DR. 2002. A practical information-theoretic approach. Model selection 
and multimodel inference, 2nd ed. Springer, New York, 2.

Carandini M, Heeger DJ. 2012. Normalization as a canonical neural computation. Nat Rev Neurosci. 13: 51- 62 .

Carrasco M. 2011. Visual attention: The past 25 years. Vision Res. 51: 1484-1525.

Chen C, Zhang X, Wang Y, Zhou T, Fang F. 2016. Neural activities in V1 create the bottom-up saliency map of natural scenes. Exp Brain Res. 234: 1769-1780.

Connor CE, Gallant JL, Preddie DC, Van Essen DC. 1996. Responses in area V4 depend on the spatial relationship between stimulus and attention. J Neurophysiol. 75: 1306-1308.

Connor CE, Preddie DC, Gallant JL, Van Essen DC. 1997. Spatial attention effects in macaque area V4. J Neurosci. 17: 3201-3214.

Corbetta M, Shulman GL. 2002. Control of goal-directed and stimulus-driven attention in the brain. Nat Rev Neurosci. 3: 201-215.

Couperus JW, Lydic KO. 2019. Attentional set and the gradient of visual spatial attention. Neurosci Lett. 712: 134495.

Dehaene S, Changeux JP. 2011. Experimental and theoretical approaches to conscious processing. Neuron 70: 200-227.

Desimone R, Duncan J. 1995. Neural mechanisms of selective visual attention. Annu Rev Neurosci. 18: 193-222.

Di Russo F, Spinelli D, Morrone MC. 2001. Automatic gain control contrast mechanisms are modulated by attention in humans: evidence from visual evoked potentials. Vision Res. 41: $2435-2447$.

Downing CJ. 1988. Expectancy and visual-spatial attention: Effects on perceptual quality. J Exp Psychol Hum Percept Perform. 14: 188-202.

Eckstein MP, Mack SC, Liston DB, Bogush L, Menzel R, Krauzlis RJ. 2013. Rethinking human visual attention: Spatial cueing effects and optimality of decisions by honeybees, monkeys and humans. Vision Res. 85: 5-19.

Eimer M. 1997. Attentional selection and attentional gradients: An alternative method for studying transient visual - spatial attention. Psychophysiology 34: 365-376.

Faivre N, Kouider S. 2011. Multi-feature objects elicit nonconscious priming despite crowding. J Vis. 11: 2. 
Fang MW, Liu T. 2019. The profile of attentional modulation to visual features. J Vis. 19: 13-13.

Fang MW, Becker MW, Liu T. 2019. Attention to colors induces surround suppression at category boundaries. Sci Rep. 9: 1443.

Fecteau JH, Munoz DP. 2006. Salience, relevance, and firing: a priority map for target selection. Trends Cogn Sci. 10: 382-390.

Finke C, Ostendorf F, Martus P, Braun M, Ploner CJ. 2008. Inhibition of orienting during a memory-guided saccade task shows a Mexican-hat distribution. Neuroscience 153: 189-195.

Frässle S, Sommer J, Jansen A, Naber M, Einhäuser W. 2014. Binocular rivalry: frontal activity relates to introspection and action but not to perception. J Neurosci. 34: 1738-1747.

Gibson BS, Kelsey EM. 1998. Stimulus-driven attentional capture is contingent on attentional set for displaywide visual features. J Exp Psychol Hum Percept Perform. 24: 699.

Handy TC, Kingstone A, Mangun GR. 1996. Spatial distribution of visual attention: Perceptual sensitivity and response latency. Percept Psychophys. 58: 613-627.

Hara Y, Pestilli F, Gardner JL. 2014. Differing effects of attention in single-units and populations are well predicted by heterogeneous tuning and the normalization model of attention. Front Computat Neurosci. 8: 12 .

Heeger DJ. 1992. Normalization of cell responses in cat striate cortex. Vis Neurosci. 9: 181-197.

Hegdé J, Felleman DJ. 2003. How selective are V1 cells for pop-out stimuli? J Neurosci. 23: 9968-9980.

Henderson JM, Macquistan AD. 1993. The spatial distribution of attention following an exogenous cue. Percept Psychophys. 53: 221-230.

Herrmann K, Montaser-Kouhsari L, Carrasco M, Heeger DJ. 2010. When size matters: attention affects performance by contrast or response gain. Nat Neurosci. 13: 1554-1559.

Hopf JM, Boehler CN, Luck SJ, Tsotsos JK, Heinze HJ, Schoenfeld MA. 2006. Direct neurophysiological evidence for spatial suppression surrounding the focus of attention in vision. Proc Natl Acad Sci USA. 103: 1053-1058.

Hopf JM, Boehler CN, Schoenfeld MA, Heinze HJ, Tsotsos JK. 2010. The spatial profile of the focus of attention in visual search: insights from MEG recordings. Vision Res. 50: $1312-1320$.

Huang L, Dobkins KR. 2005. Attentional effects on contrast discrimination in humans: evidence 
for both contrast gain and response gain. Vision Res. 45: 1201-1212.

Huang L, Wang L, Shen W, Li M, Wang S, Wang X, Ungerleider LG, Zhang X. 2020. A source for awareness-dependent figure-ground segregation in human prefrontal cortex. Proc Natl Acad Sci USA. 117: 30836-30847.

Hsieh PJ, Colas JT, Kanwisher N. 2011. Pop-out without awareness: Unseen feature singletons capture attention only when top-down attention is available. Psychol Sci. 22: 1220-1226.

Itthipuripat S, Garcia JO, Rungratsameetaweemana N, Sprague TC, Serences JT. 2014. Changing the spatial scope of attention alters patterns of neural gain in human cortex. J Neurosci. 34: $112-123$.

Itthipuripat S, Sprague TC, Serences JT. 2019. Functional MRI and EEG index complementary attentional modulations. J Neurosci. 39: 6162-6179.

Jazayeri M, Movshon JA. 2006. Optimal representation of sensory information by neural populations. Nat Neurosci. 9: 690-696.

Jiang Y, Costello P, Fang F, Huang M, He S. 2006. A gender- and sexual orientation-dependent spatial attentional effect of invisible images. Proc Natl Acad Sci USA 103: 17048-17052.

Kastner S, Nothdurft HC, Pigarev IN. 1997. Neuronal correlates of pop-out in cat striate cortex. Vision Res. 37: 371-376.

Kastner S, Ungerleider LG. 2000. Mechanisms of visual attention in the human cortex. Annu Rev Neurosci. 23: 315-341.

Kentridge RW, Heywood CA, Weiskrantz L. 1999a. Attention without awareness in blindsight. Proc Biol Sci. 266: 1805-1811.

Kentridge RW, Heywood CA, Weiskrantz L. 1999b. Effects of temporal cueing on residual visual discrimination in blindsight. Neuropsychologia 37: 479-483.

Kentridge RW, Heywood CA, Weiskrantz L. 2004. Spatial attention speeds discrimination without awareness in blindsight. Neuropsychologia 42: 831-835.

Kim YJ, Grabowecky M, Paller KA, Muthu K, Suzuki S. 2007. Attention induces synchronization-based response gain in steady-state visual evoked potentials. Nat Neurosci. 10: $117-125$.

Koch C, Massimini M, Boly M, Tononi G. 2016. Neural correlates of consciousness: progress and problems. Nat Rev Neurosci. 17: 307-321. 
Koch C, Ullman S. 1985. Shifts in selective visual attention: towards the underlying neural circuitry. Hum Neurobiol. 4: 219-227.

LaBerge D. 1983. Spatial extent of attention to letters and words. J Exp Psychol Hum Percept Perform. 9: 371-379.

Lau H, Rosenthal D. 2011. Empirical support for higher-order theories of conscious awareness. Trends Cogn Sci. 15: 365-373.

Lee J, Maunsell JH. 2009. A normalization model of attentional modulation of single unit responses. PLoS One 4: e4651.

Lee J, Maunsell JH. 2010. The effect of attention on neuronal responses to high and low contrast stimuli. J Neurophysiol. 104: $960-971$.

Li X, Lu ZL, Tjan BS, Dosher BA, Chu W. 2008. Blood oxygenation level-dependent contrast response functions identify mechanisms of covert attention in early visual areas. Proc Natl Acad Sci USA 105: 6202-6207.

Lin JY, Murray SO, Boynton GM. 2009. Capture of attention to threatening stimuli without perceptual awareness. Curr Biol. 19: 1118-1122.

Ling S, Carrasco M. 2006. Sustained and transient covert attention enhance the signal via different contrast response functions. Vision Res. 46: 1210-1220.

Mangun GR, Hillyard SA. 1988. Spatial gradients of visual attention: behavioral and electrophysiological evidence. Electroencephalogr Clin Neurophysiol. 70: 417-428.

Martínez-Trujillo J, Treue S. 2002. Attentional modulation strength in cortical area MT depends on stimulus contrast. Neuron 35: 365-370.

Mashour GA, Roelfsema P, Changeux JP, Dehaene S. 2020. Conscious processing and the global neuronal workspace hypothesis. Neuron 105: 776-798.

Mazer JA, Gallant JL. 2003. Goal-related activity in V4 during free viewing visual search. Evidence for a ventral stream visual salience map. Neuron 40: 1241-1250.

McAdams CJ, Maunsell JH. 1999. Effects of attention on orientation tuning functions of single neurons in macaque cortical area V4. J Neurosci. 19: 431-441.

Montaser-Kouhsari L, Rajimehr R. 2005. Subliminal attentional modulation in crowding condition. Vision Res. 45: 839-844.

Moore T, Zirnsak M. 2017. Neural mechanisms of selective visual attention. Annu Rev Psychol. 
68: $47-72$.

Moran J, Desimone R. 1985. Selective attention gates visual processing in the extrastriate cortex. Science 229: 782-784.

Morrone MC, Denti V, Spinelli D. 2002. Color and luminance contrasts attract independent attention. Curr Biol. 12: 1134-1137.

Mounts JR. 2000. Evidence for suppressive mechanisms in attentional selection: Feature singletons produce inhibitory surrounds. Percept Psychophys. 62: 969-983.

Mulckhuyse M, Theeuwes J. 2010. Unconscious attentional orienting to exogenous cues: A review of the literature. Acta Psychol. 134: 299-309.

Müller NG, Kleinschmidt A. 2004. The attentional 'spotlight's' penumbra: Center-surround modulation in striate cortex. Neuroreport 15: 977-980.

Müller NG, Mollenhauer M, Rosler A, Kleinschmidt A. 2005. The attentional field has a Mexican hat distribution. Vision Res. 45: 1129-1137.

Murray SO. 2008. The effects of spatial attention in early human visual cortex are stimulus independent. J Vis. 8: 1-11.

Naccache L, Blandin E, Dehaene S. 2002. Unconscious masked priming depends on temporal attention. Psychol Sci. 13: 416-424.

Naka KI, Rushton WA. 1966. S-potentials from luminosity units in the retina of fish (Cyprinidae). J Physiol. 185: 587-599.

Nakayama K, Mackeben M. 1989. Sustained and transient components of focal visual attention. Vision Res. 29: 1631-1647.

Pestilli F, Carrasco M, Heeger DJ, Gardner JL. 2011. Attentional enhancement via selection and pooling of early sensory responses in human visual cortex. Neuron 72: 832-846.

Pestilli F, Ling S, Carrasco M. 2009. A population-coding model of attention's influence on contrast response: estimating neural effects from psychophysical data. Vision Res. 49: $1144-1153$.

Posner MI. 2016. Orienting of attention: Then and now. Q J Exp Psychol. 69: 1864-1875.

Posner MI, Snyder CRR, Davidson BJ. 1980. Attention and the detection of signals. J Exp Psychol. 109: 160-174.

Raftery AE. 1999. Bayes factors and BIC. Sociol Methods Res. 27: 411-417. 
Reynolds JH, Chelazzi L. 2004. Attentional modulation of visual processing. Annu Rev Neurosci. 27: 611-647.

Reynolds JH, Chelazzi L, Desimone R. 1999. Competitive mechanisms subserve attention in macaque areas V2 and V4. J Neurosci. 19: 1736-1753.

Reynolds JH, Heeger DJ. 2009. The normalization model of attention. Neuron 61: 168-185.

Reynolds JH, Pasternak T, Desimone R. 2000. Attention increases sensitivity of V4 neurons. Neuron 26: 703-714.

Robertson CE, Kravitz DJ, Freyberg J, Baron-Cohen S, Baker CI. 2013. Tunnel vision: sharper gradient of spatial attention in autism. J Neurosci. 33: 6776-6781.

Schall JD, Hanes DP. 1993. Neural basis of saccade target selection in frontal eye field during visual search. Nature 366: 467-469.

Schall JD, Sato TR, Thompson KG, Vaughn AA, Juan CH. 2004. Effects of search efficiency on surround suppression during visual selection in frontal eye field. J Neurophysiol. 91: $2765-2769$.

Schallmo MP, Kolodny T, Kale AM, Millin R, Flevaris AV, Edden RA, Gerdts J, Bernier RA, Murray SO. 2020. Weaker neural suppression in autism. Nat Commun. 11: 1-13.

Schmitz TW, Duncan J. 2018. Normalization and the cholinergic microcircuit: a unified basis for attention. Trends Cogn Sci. 22: 422-437.

Schwarz G. 1978. Estimating the dimension of a model. Annu Stat. 6: 461-464.

Schwedhelm P, Krishna BS, Treue S. 2016. An extended normalization model of attention accounts for feature-based attentional enhancement of both response and coherence gain. PLoS Comput Biol. 12: e1005225.

Serences JT, Yantis S. 2007. Spatially selective representations of voluntary and stimulus-driven attentional priority in human occipital, parietal, and frontal cortex. Cereb Cortex 17: $284-293$.

Sergent C, Wyart V, Babo-Rebelo M, Cohen L, Naccache L, Tallon-Baudry C. 2013. Cueing attention after the stimulus is gone can retrospectively trigger conscious perception. Curr Boil. 23: $150-155$.

Shipp S. 2004. The brain circuitry of attention. Trends Cogn Sci. 8: 223-230.

Shulman GL, Wilson J, Sheehy JB. 1985. Spatial determinants of the distribution of attention. 
Percept Psychophys. 37: 59-65.

Squire RF, Noudoost B, Schafer RJ, Moore T. 2013. Prefrontal contributions to visual selective attention. Annu Rev Neurosci. 36: 451-466.

Tkacz-Domb S, Yeshurun Y. 2018. The size of the attentional window when measured by the pupillary response to light. Sci Rep. 8: 1-7.

Tononi G, Boly M, Massimini M, Koch C. 2016. Integrated information theory: from consciousness to its physical substrate. Nat Rev Neurosci. 17: 450-461.

Tsotsos JK, Culhane SM, Wai WYK, Lai Y, Davis N, Nuflo F. 1995. Modeling visual attention via selective tuning. Artif Intell. 78: 507-545.

Tsotsos JK, Rodriguez-Sanchez AJ, Rothenstein AL, Simine E. 2008. The different stages of visual recognition need different attentional binding strategies. Brain Res. 1225: 119-132.

Tsuchiya N, Wilke M, Frässle S, Lamme VA. 2015. No-report paradigms: extracting the true neural correlates of consciousness. Trends Cogn Sci. 19: 757-770.

Wagenmakers EJ. 2007. A practical solution to the pervasive problems ofp values. Psychon Bull Rev. 14: 779-804.

Williford T, Maunsell JH. 2006. Effects of spatial attention on contrast response functions in macaque area V4. J Neurophysiol. 96: 40-54.

Yantis S, Jonides J. 1984. Abrupt visual onsets and selective attention: evidence from visual search. J Exp Psychol Hum Percept Perform. 10: 601.

Yeshurun Y. 2019. The spatial distribution of attention. Curr Opin Psychol. 29: 76-81.

Zhang X, Fang F. 2012. Object-based attention guided by an invisible object. Exp Brain Res. 223: $397-404$

Zhang X, Japee S, Safiullah Z, Mlynaryk N, Ungerleider LG. 2016. A normalization framework for emotional attention. PLoS Biol. 14: e1002578.

Zhang X, Zhaoping L, Zhou T, Fang F. 2012. Neural activities in V1 create a bottom-up saliency map. Neuron 73: 183-192. 


\section{Figure Legends}
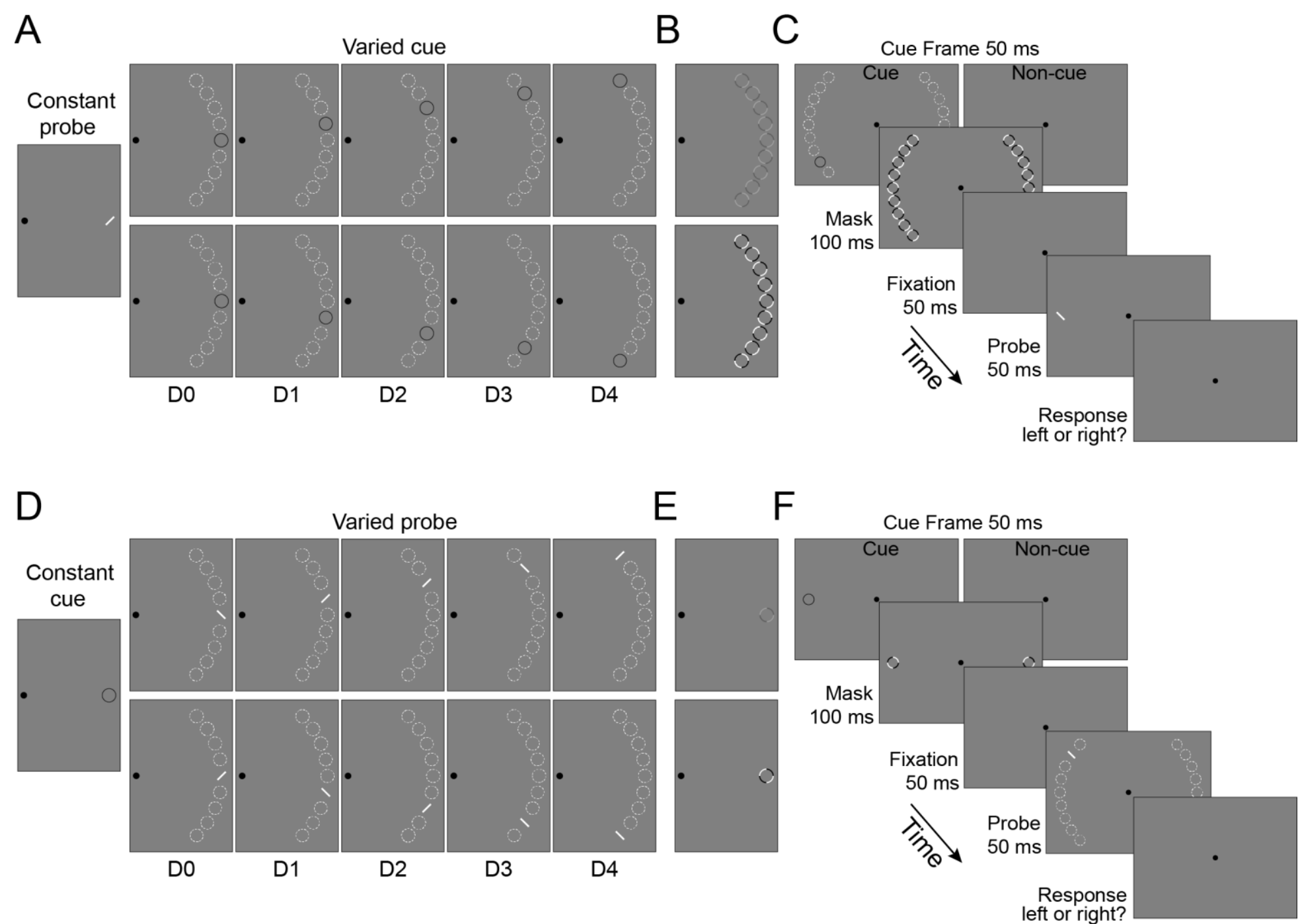

Figure 1. Stimuli and Psychophysical Protocol of Distribution Experiments. Each

texture stimulus contained 18 positions, indicating by the dashed white circles (not displayed during the experiments), settled at an iso-eccentric distance from fixation (the black dot); a half of them were located in the left visual field and the other half was located in the right visual field. During Experiment 1 (A), in each visual field (in this case, the right visual field), the probe, a tilted line (left), always appeared at the center of 9 positions while the exogenous cue, a low-luminance ring (right), appeared equiprobably and randomly at one of the 9 possible positions in the cue condition 
(83.33\% of trials and $16.67 \%$ of trails for each distance) and was absent in the non-cue condition (16.67\% of trials). The probe position was constant and the cue position varied, thus there were five possible distances between them, ranging from D0 (cue and probe at the same location) through D4 (cue and probe four items away from each other). During Experiment 2 (D), in each visual field (in this case, the right visual field), the exogenous cue appeared (the cue condition, 50\% of trials) or was absent (the non-cue condition, $50 \%$ of trials) in the center of 9 positions. The probe appeared equiprobably and randomly at one of the 9 possible positions. The cue position was constant and the probe position varied, there were also five possible distances between them ( $20 \%$ of trials for each distance), ranging from D0 through D4. Low- (top) and high- (bottom) contrast masks used for the visible and invisible conditions, respectively, in Experiments 1 (B) and 2 (E). Psychophysical protocol of Experiments 1 (C) and 2 (F). A cue frame with (the cue condition) or without (the non-cue condition) exogenous cue was presented for $50-\mathrm{ms}$, followed by a $100-\mathrm{ms}$ mask (low- and high-contrast for visible and invisible conditions, respectively) and another 50-ms fixation interval. Then a probe line, orientating at $45^{\circ}$ or $135^{\circ}$ away from the vertical, was presented for 50-ms. Subjects were asked to press one of two buttons as rapidly and correctly as possible to indicate the orientation of the probe $\left(45^{\circ}\right.$ or $\left.135^{\circ}\right)$ 
bioRxiv preprint doi: https://doi.org/10.1101/2021.04.18.440351; this version posted April 20, 2021. The copyright holder for this preprint (which was not certified by peer review) is the author/funder, who has granted bioRxiv a license to display the preprint in perpetuity. It is made available under aCC-BY 4.0 International license.

A

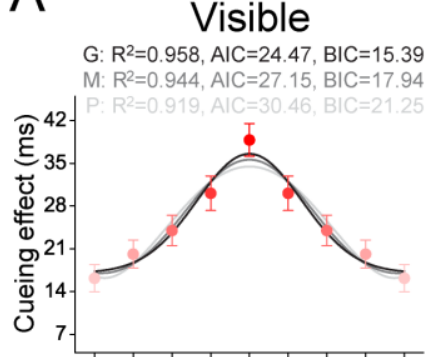

G: $R^{2}=0.976, A I C=24.35, B I C=15.14$

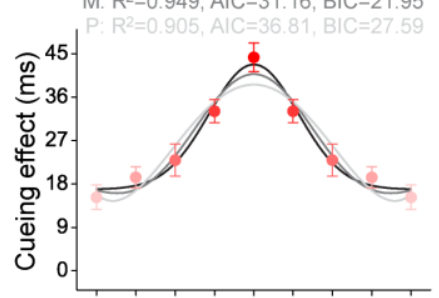

$G: R^{2}=0.968, A I C=24.58, B I C=15.37$ $M: R^{2}=0.947, A I C=29.34, B I C=20.03$

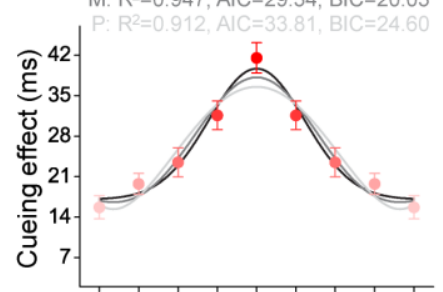

D4 D3 D2 D1 D0 D1 D2 D3 D4

$\mathrm{D}$

G: $R^{2}=0.974, A I C=15.28, B I C=6.07$ $M: R^{2}=0.952, A I C=20.60, B I C=11.39$

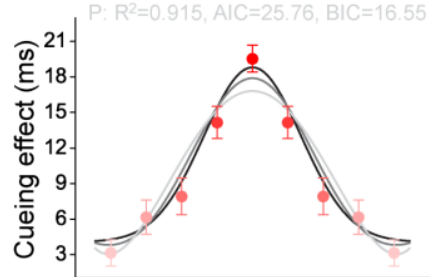

$\mathrm{G}: \mathrm{R}^{2}=0.948, \mathrm{AIC}=22.61, \mathrm{BIC}=13.40$ $M: R^{2}=0.928, A I C=25.51, B I C=16.30$

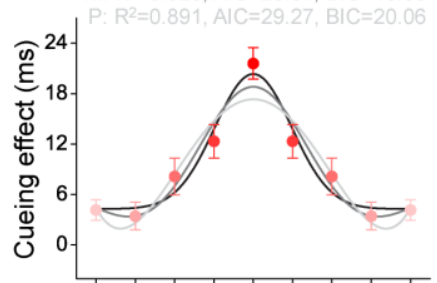

$\mathrm{G}: \mathrm{R}^{2}=0.978, \mathrm{AIC}=14.10, \mathrm{BIC}=4.89$ $\mathrm{M}: \mathrm{R}^{2}=0.956, \mathrm{AIC}=20.31, \mathrm{BIC}=11.10$

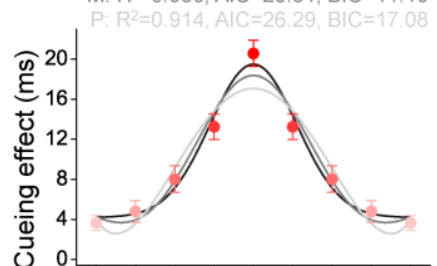

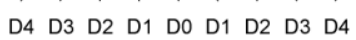

Invisible

G: $R^{2}=0.988, A I C=3.93, B I C=-5.28$ $M: R^{2}=0.973, A I C=11.61, B I C=2.40$

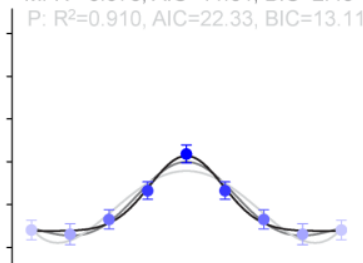

$G: R^{2}=0.990, A I C=13.08, B I C=3.87$ $M: R^{2}=0.821, A I C=39.33, B I C=30.12$

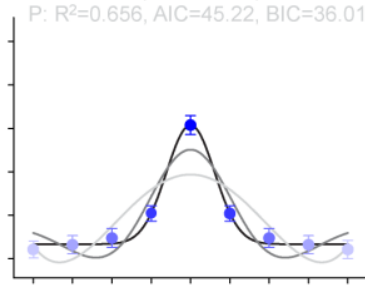

$G: R^{2}=0.988, A I C=10.44, B I C=1.23$

$\mathrm{M}: \mathrm{R}^{2}=0.883, \mathrm{AIC}=30.70, \mathrm{BIC}=21.49$

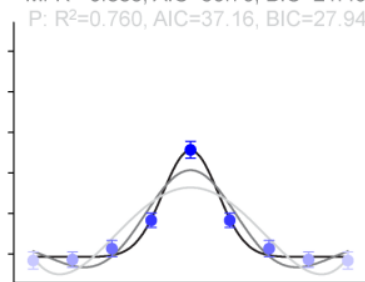

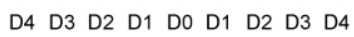

G: $R^{2}=0.943, A I C=3.93, B I C=-5.28$ $M: R^{2}=0.727, A I C=30.38, B I C=21.17$

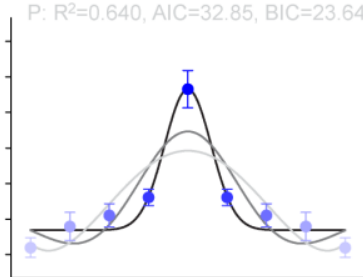

$G: R^{2}=0.956, A I C=24.05, B I C=14.84$ $\mathrm{M}: \mathrm{R}^{2}=0.737, \mathrm{AIC}=40.08, \mathrm{BIC}=30.87$

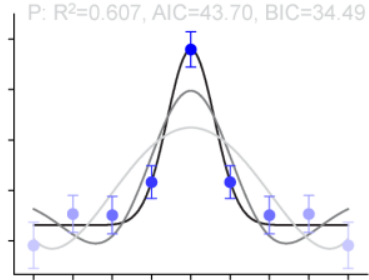

$G: R^{2}=0.953, A I C=20.28, B I C=11.07$ $M: R^{2}=0.732, A I C=35.92, B I C=26.70$

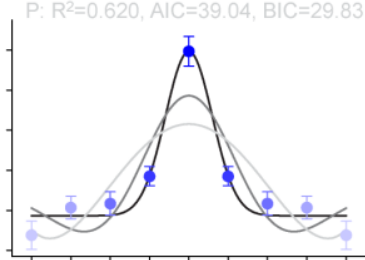

D4 D3 D2 D1 D0 D1 D2 D3 D4
B
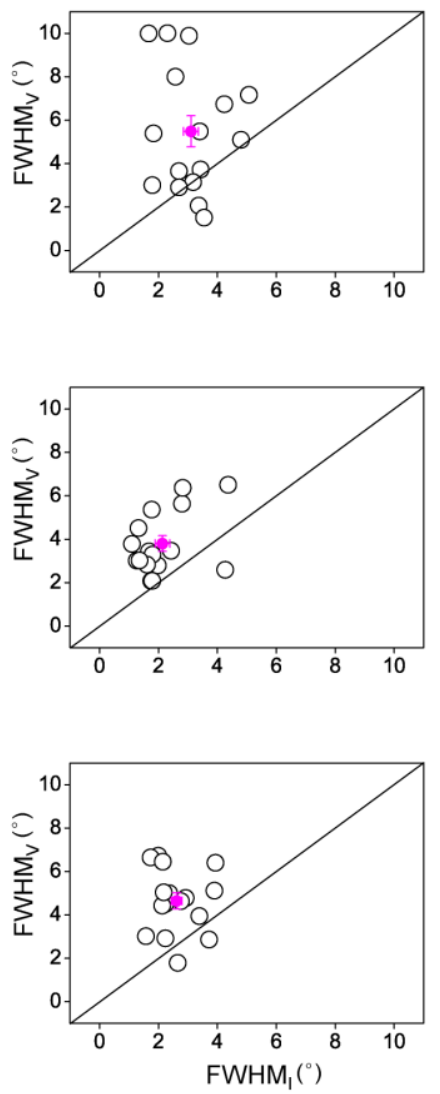

$E$
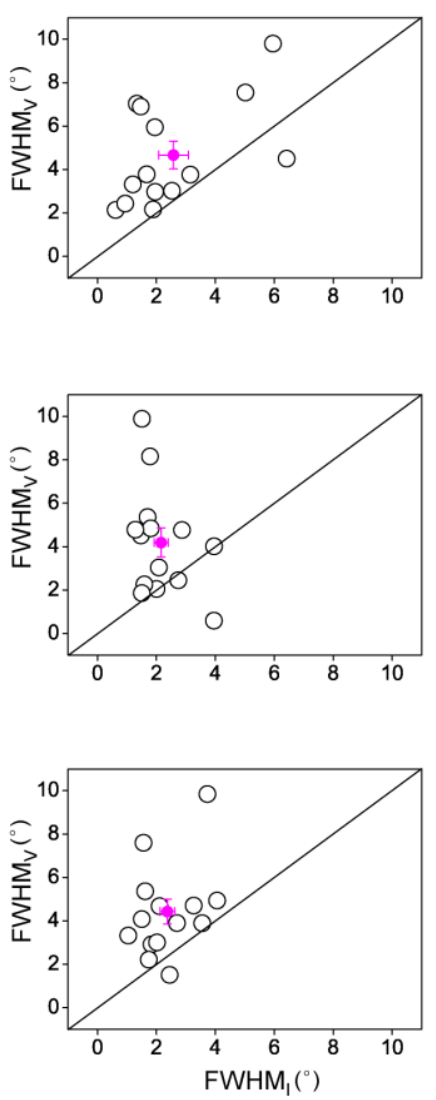

50
C

Peak: $r=-0.294, p=0.269$

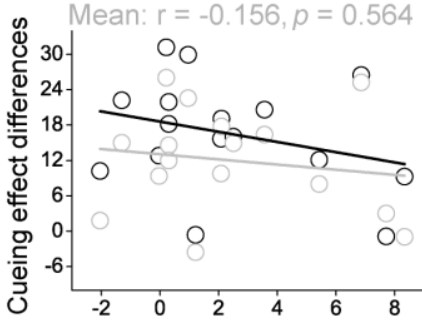

Peak: $r=0.428, p=0.098$

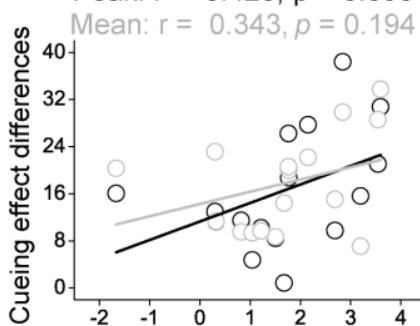

Peak: $r=0.0484, p=0.859$

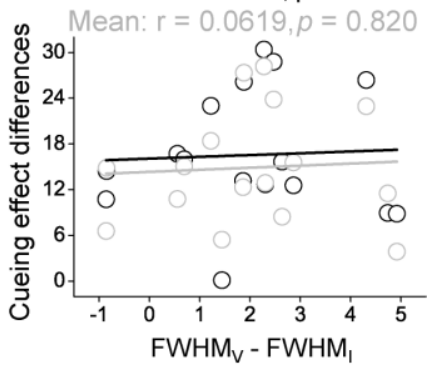

F Peak: $r=0.227, p=0.434$

\& Mean: $r=0.334, p=0.243$

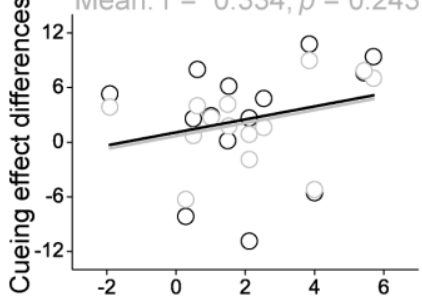

Peak: $r=0.094, p=0.749$

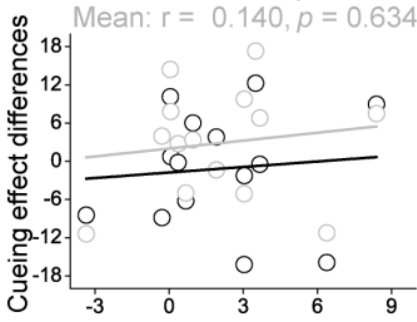

Peak: $r=0.151, p=0.607$

\& Mean: $r=0.235, p=0.419$

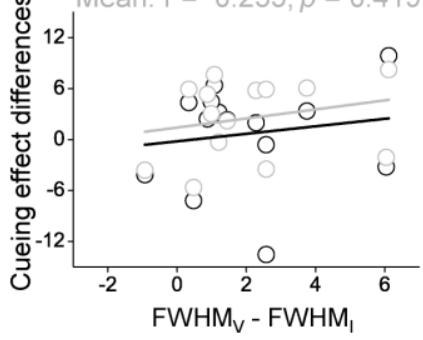


Figure 2. Results of Distribution Experiments. (A) and (D) The cueing effect of each distance (D0 to D4) in visible (left) and invisible (right) conditions for Experiment 1 (top), Experiment 2 (middle), and Experiments $1 \& 2$ (bottom), and the best fitting monotonic Gaussian function and two non-monotonic functions (Mexican Hat and Polynomial) to these cueing effects across distances. Each cueing effect was quantified as the difference between the reaction time of the probe task performance in the non-cue condition and that in the cue condition. Error bars denote 1 SEM calculated across subjects. G: Gaussian model; M: Mexican Hat model; P: Polynomial model; R²: R-squared; AIC: Akaike Information Criterion; BIC: Bayesian Information Criterion. (B) and (E) The fitted FWHM bandwidth of monotonic Gaussian model in visible and invisible conditions for Experiment 1 (top), Experiment 2 (middle), and Experiments $1 \& 2$ (bottom). FWHM $\mathrm{V}$ and $\mathrm{FWHM}_{\mathrm{I}}$ : the fitted FWHM bandwidth of the Gaussian model in the visible and invisible conditions, respectively. Open symbols indicate individual subjects and a filled symbol indicate mean across subjects. Error bars denote 1 SEM calculated across subjects. (C) and (F) Correlations between the increased FWHM bandwidth and peak cueing effect (i.e., the cueing effect of D0, gray), and between the increased FWHM bandwidth and mean cueing effect (black) across distances, in the visible condition relative to the invisible condition, across individual subjects, in Experiment 1 (top), Experiment 2 (middle), and Experiments 1 $\& 2$ (bottom). 
A
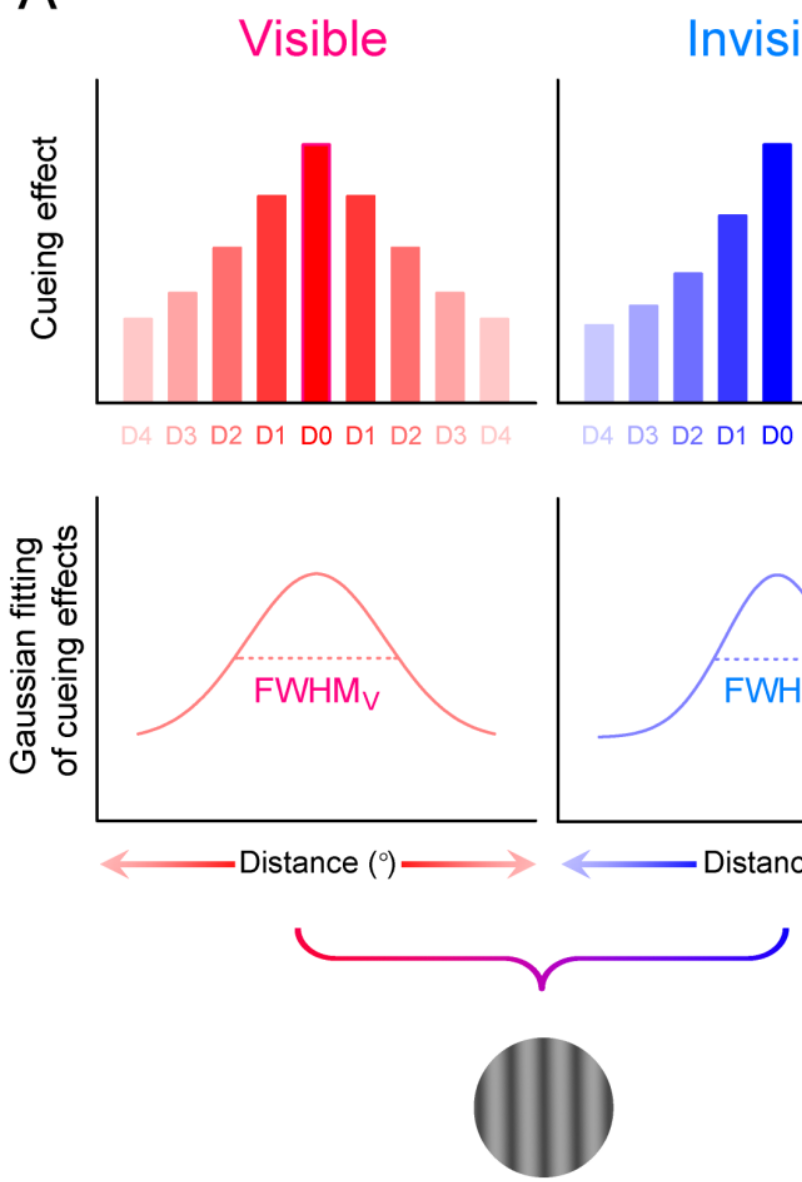

$$
D=\frac{F W H M_{V}+F W H M_{I}}{2}
$$

B

Contrast gain

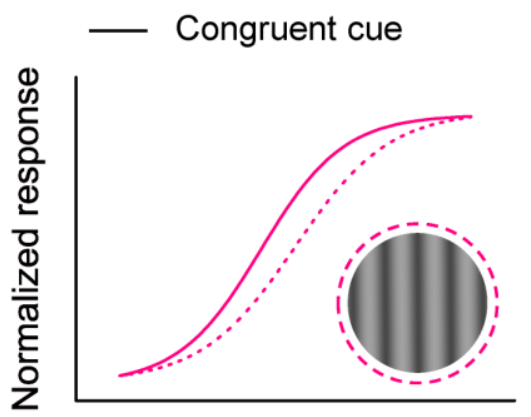

Contrast

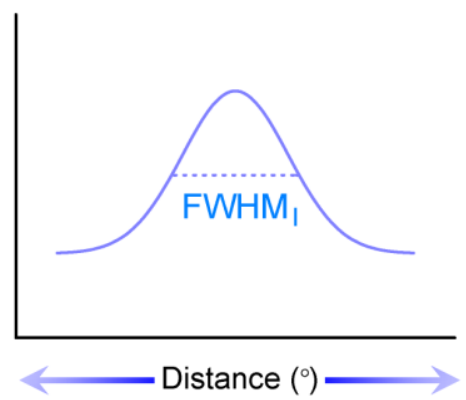

Response gain

Invisible

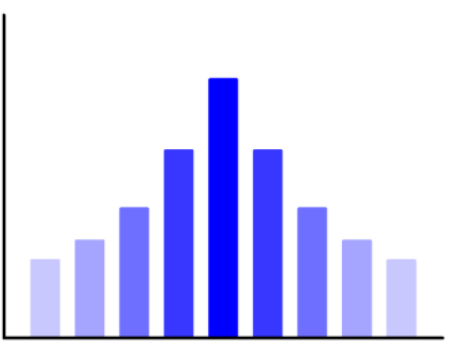

D4 D3 D2 D1 D0 D1 D2 D3 D4

-- Incongruent cue

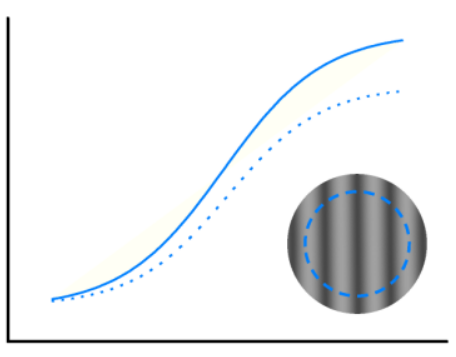

Contrast
C

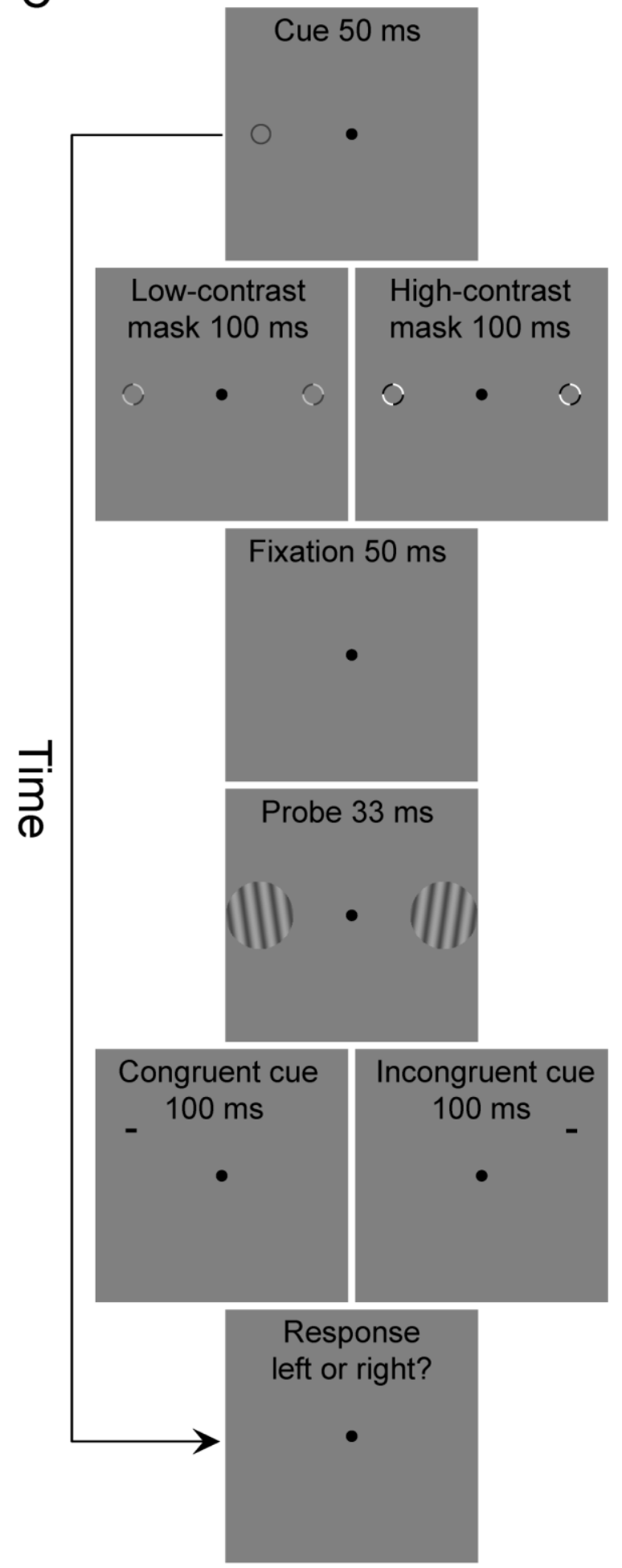

Figure 3. The Normalization Model of Attention Modulated by Awareness and

Psychophysical Protocol in Normalization Experiments. (A) An example of grating probes manipulated from Distribution Experiments. For each subject, the diameter of grating $=\left(F W H M_{V+} F W H M_{I}\right) / 2$, where $F W H M_{V}$ and $F W H M_{I}$ are their fitted FWHM 
bandwidth of the Gaussian model with and without awareness during Distribution Experiments (Experiments $1 \&$ 2), respectively. (B) Relative to the stimulus size, the attention field was broadened by visible exogenous cues (left). Under this configuration, the normalization model predicts a contrast-gain shift, with the largest effects occurring at mid-contrasts and little to no effect at low and high contrasts. Relative to the stimulus size, the attention field was narrowed by invisible cues (right). Under this configuration, the normalization model predicts a response-gain shift, with the largest effects occurring at high contrasts and little to no effect at low and mid-contrasts. The dashed circles indicate simulated attention field size. (C) Psychophysical protocol. The exogenous cue randomly appeared at the center of 9 positions in left or right hemifield with equal probability, followed by a $100-\mathrm{ms}$ mask (low- and high-contrast for visible and invisible conditions, respectively) and another 50-ms fixation interval. Then, a pair of gratings (with identical contrasts) was presented for $33 \mathrm{~ms}$ in the left and right hemifields, one of which was the target. Subjects were asked to press one of two buttons to indicate the orientation of the target grating (leftward or rightward tilted) and received auditory feedback if their response was incorrect. The target grating was indicated by a peripheral $100 \mathrm{~ms}$ response cue above one of the grating locations, but not at the grating location to avoid masking. A congruent cue was defined as a match between the exogenous cue location and response cue location (half the trials); an incongruent cue was defined as a mismatch (half the trials). 


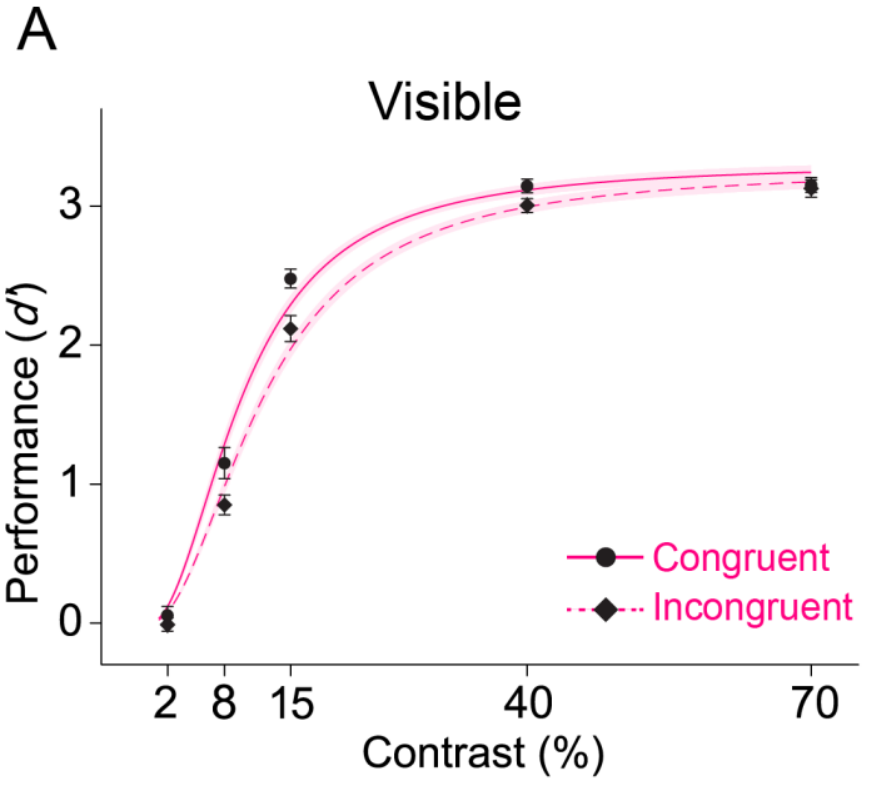

B
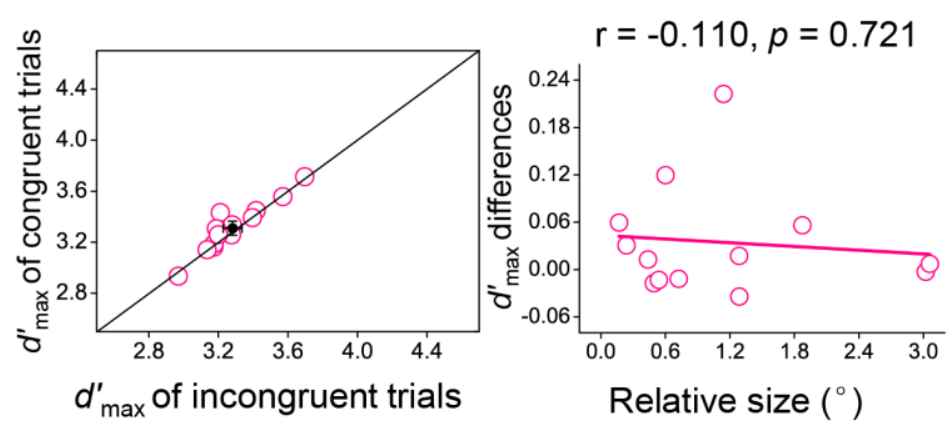

D

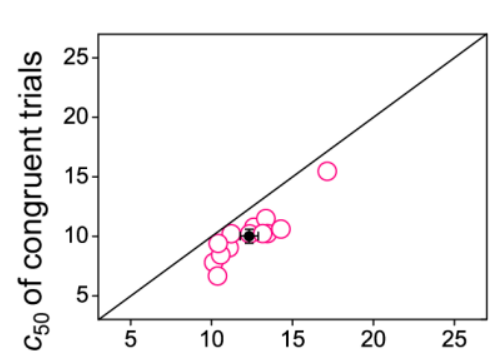

$c_{50}$ of incongruent trials

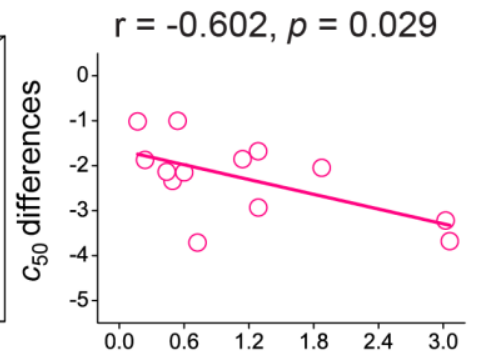

Relative size $\left({ }^{\circ}\right)$

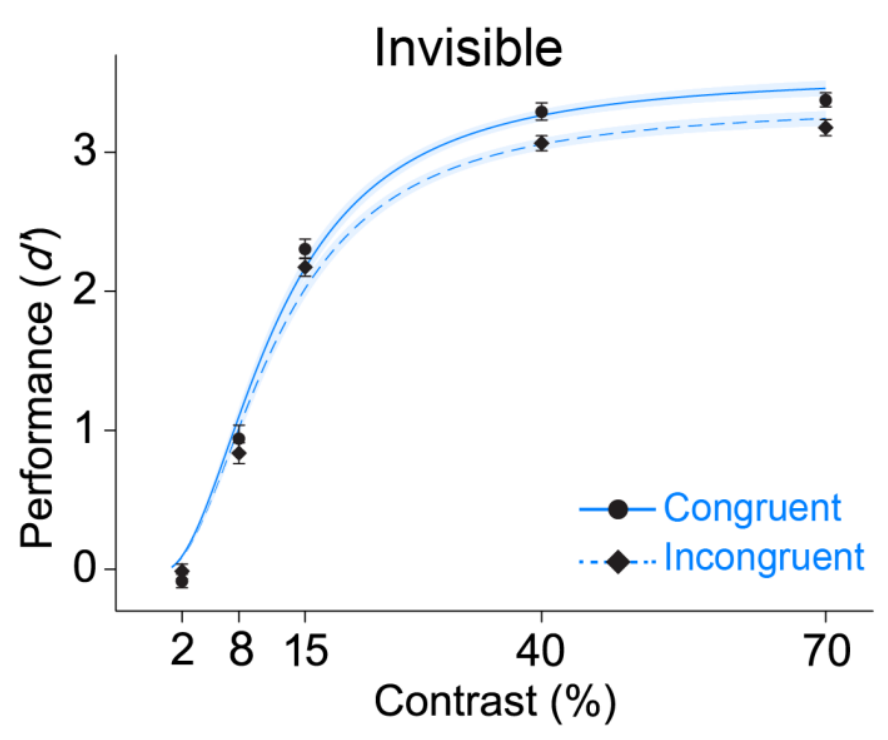

C
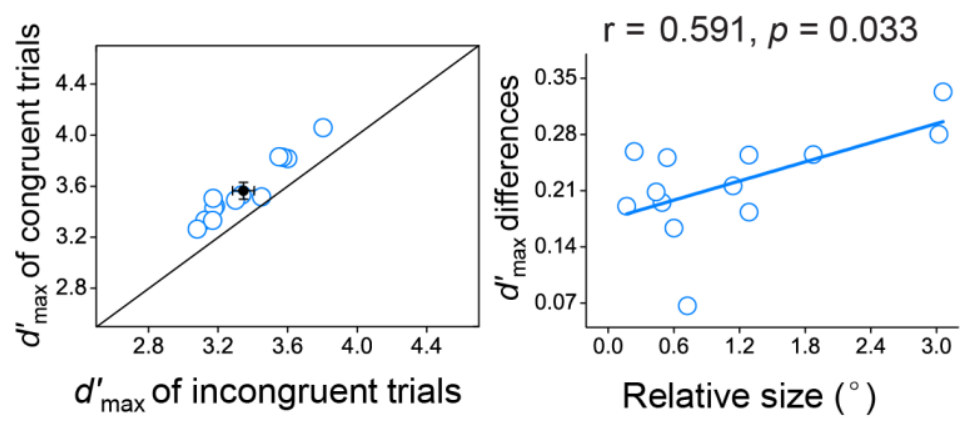

$E$

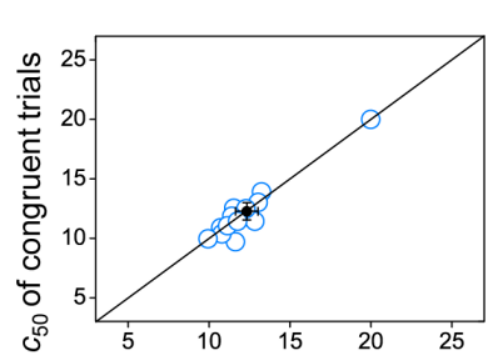

$c_{50}$ of incongruent trials

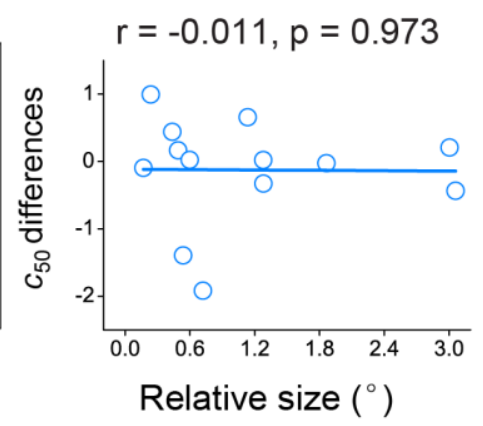

Figure 4. Effects of Visible and Invisible Cues on Performance (d') as a Function of

Contrast. (A) Mean $d^{\prime}$ plotted as psychometric functions of stimulus contrast and awareness (visible, left; invisible, right) for congruent and incongruent trials. Error bars denote 1 SEM calculated across subjects. $d_{\text {max }}^{\prime}$ for congruent and incongruent trials in the visible $(\mathbf{B})$ and invisible $(\mathbf{C})$ conditions, and correlations between the 
relative size of attention field to the stimulus [i.e., $\left(\mathrm{FWHM}_{\mathrm{V}}-\mathrm{FWHM}_{\mathrm{I}}\right) / 2$, where FWHM $_{\mathrm{V}}$ and FWHM $\mathrm{I}$ are the fitted FWHM bandwidth of the Gaussian model for the visible and invisible conditions, respectively] and the $d_{\max }^{\prime}$ differences between congruent and incongruent trials across individual subjects. Open symbols indicate individual subjects and a filled symbol indicate mean across subjects. Error bars denote 1 SEM calculated across subjects. $d_{\max }^{\prime}$ : asymptotic performance at high contrast levels. $c_{50}$ for congruent and incongruent trials in the visible (D) and invisible (E) conditions, and correlations between the relative size of attention field to the stimulus and the $c_{50}$ differences between congruent and incongruent trials across individual subjects. Open symbols indicate individual subjects and a filled symbol indicate mean across subjects. Error bars denote 1 SEM calculated across subjects. $c_{50}$ : the contrast yielding half maximum performance. 
A
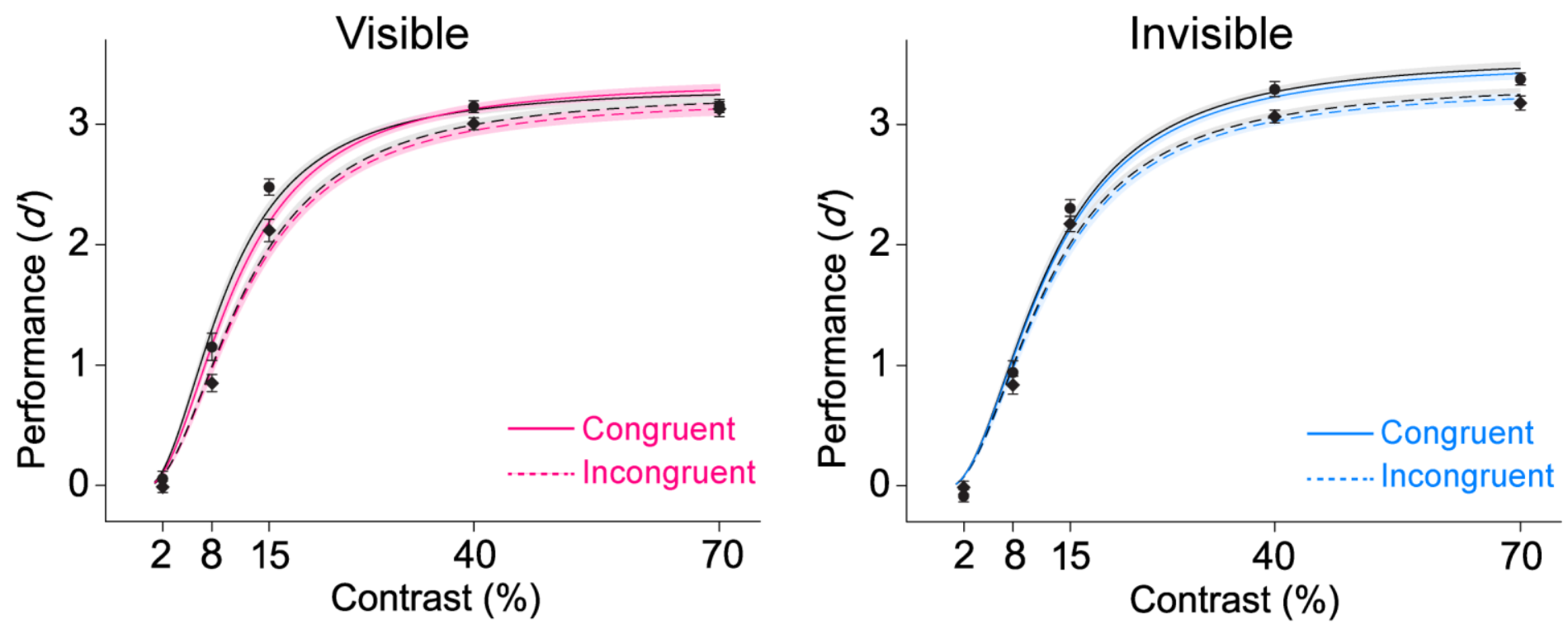

B

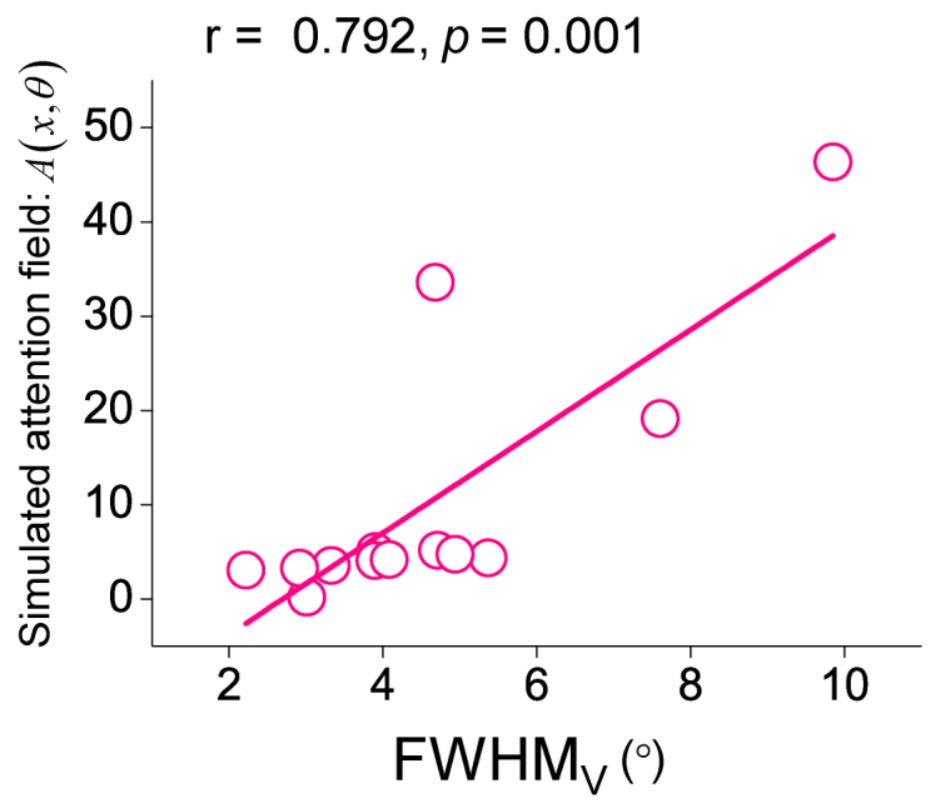

Figure 5. Model Predictions of Contrast Response Functions for the Normalization

Model of Attention. (A) Data points with plus/minus one standard error are the mean performance $\left(d^{\prime}\right)$ across subjects for awareness (visible and invisible), cue validity (congruent and incongruent), and stimulus contrasts. The fitted performances $\left(d^{\prime}\right)$ using the standard Naka-Rushton equation and the original normalization model of 
attention with 4 free parameters are indicated by the black and colored lines, respectively. (B) Correlations between the simulated attention field $[A(x, \theta)]$ of the original normalization model of attention with 4 free parameters and the experimental attention field in the visible (left) and invisible (right) conditions, across individual subjects. Note that the simulated attention fields $[A(x, \theta)]$ are in arbitrary units; only the relative values are meaningful. 
bioRxiv preprint doi: https://doi.org/10.1101/2021.04 18.440351; this version posted April 20, 2021. The copyright holder for this preprint

(which was not certified by peer review) is the author/funder, who has granted bioRxiv a license to display the preprint in perpetuity. It is made available under aCC-BY 4.0 International license.

\section{Supplementary data}

Figure S1. Protocol and Results of the Two-alternative Forced-choice Test

Figure S2. False alarm, Miss, and Removal Rates in Distribution Experiments

Figure S3. Eye Movement Data in Distribution and Normalization Experiments

Figure S4. Normalized $\mathrm{R}^{2}$ of Individual Subjects in Distribution Experiments

Figure S5. Contrast Response Functions of Individual Subjects in Normalization

Experiments 
A

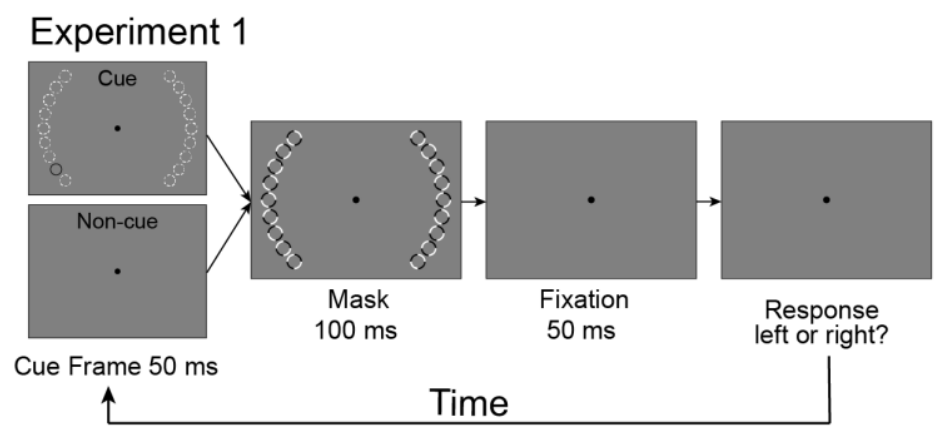

\section{Experiment 2}

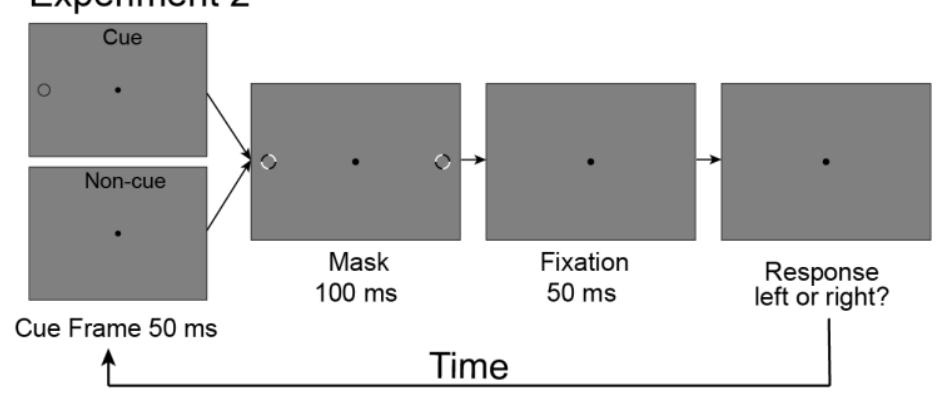

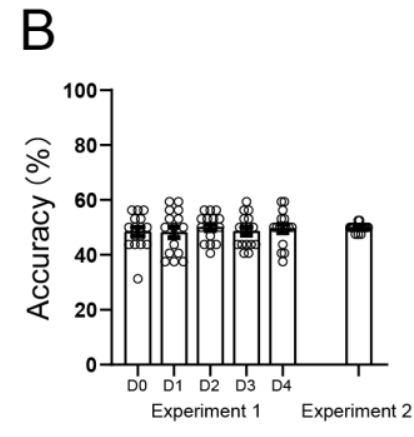
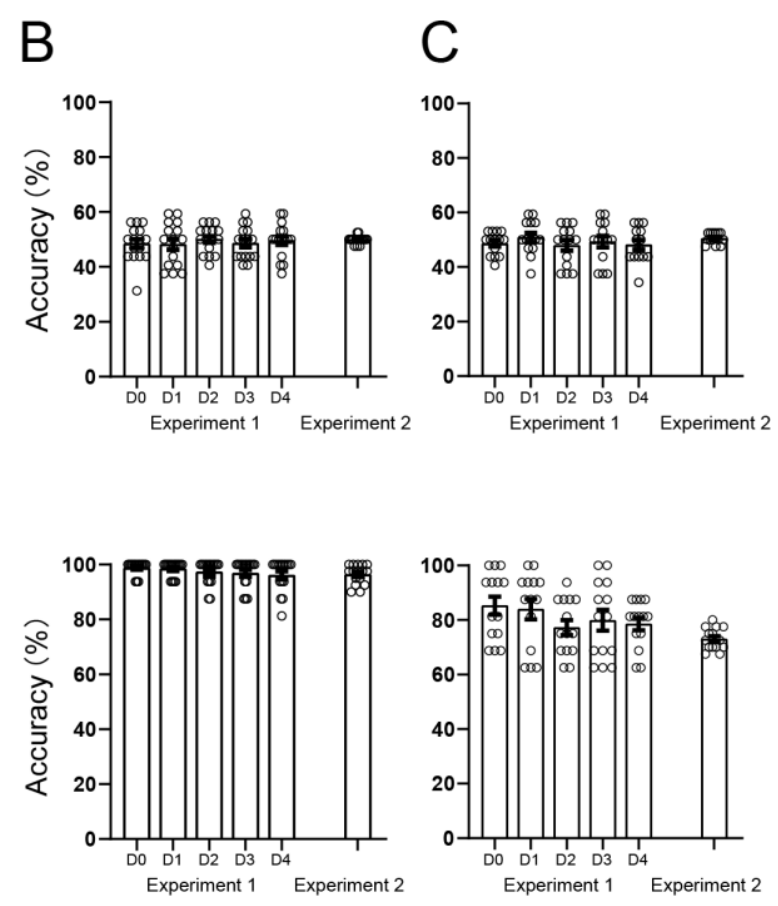

Figure S1. Protocol and Results of the Two-alternative Forced-choice Test. (A) The stimuli and procedure in the two-alternative forced-choice (2AFC) experiment (i.e., Experiment 3) were the similar to those in Experiments 1 (top) and 2 (bottom), except that no probe was presented. Experiment 3 checked the effectiveness of the awareness manipulation in Experiments 1 and 2, and was always before them. In Experiment 3, all subjects underwent a 2AFC task to determine whether the masked cue was visible or invisible in a criterion-free way. After the presentation of a masked cue frame, subjects were asked to indicate which side (left or right) from the fixation they thought the exogenous cue appeared. Their performances were significantly higher or not statistically different from chance, providing an objective confirmation that the exogenous cue was indeed visible or invisible to subjects, respectively. (B) For the invisible condition (top), subjects reported that they were unaware of the exogenous cue and could not detect which visual filed contained it. Their performances were not 
statistically different from chance [mean percent correct \pm standard error of the mean, Experiment 1 (i.e., varied cue), D0: $47.656 \pm 1.651 \%$, D1: $48.242 \pm 1.996 \%$, D2: $49.609 \pm 1.303 \%, \mathrm{D} 3: 45.508 \pm 2.096 \%, \mathrm{D} 4: 49.609 \pm 1.533 \%$, all $\mathrm{t}_{15}<0.968, p>$ $0.348, \eta_{\mathrm{p}}^{2}<0.500$; Experiment 2 (i.e., constant cue): $49.503 \pm 0.411 \%, \mathrm{t}_{15}=0.436, p=$ $\left.0.669, \eta_{\mathrm{p}}^{2}=0.225\right]$; for the visible condition, by contrast, their performance was significantly higher than chance (Experiment 1, D0: $98.828 \pm 0.630 \%, \mathrm{D} 1: 98.438 \pm$ 0.699\%, D2: $97.266 \pm 1.137 \%$, D3: $96.875 \pm 1.276 \%$, D4: $96.094 \pm 1.496 \%$, all $\mathrm{t}_{15}>$ 30.812, $p<0.001, \eta_{\mathrm{p}}^{2}>15.911$; Experiment 2: $99.503 \pm 0.411 \%, \mathrm{t}_{15}=52.557, p<$ 0.001, $\eta_{\mathrm{p}}^{2}=27.140$ ). Furthermore, for the Experiment 1, subjects' performances were submitted to a repeated-measures ANOVA with awareness (visible and invisible) and distance (D0 to D4) as within-subjects factors. The main effect of distance $\left(\mathrm{F}_{4,60}=\right.$ 0.215, $\left.p=0.929, \eta_{\mathrm{p}}^{2}=0.014\right)$ and the interaction between the two factors $\left(\mathrm{F}_{4,60}=\right.$ 0.942, $\left.p=0.446, \eta_{\mathrm{p}}^{2}=0.059\right)$ were not significant, but the main effect of awareness was significant $\left(\mathrm{F}_{1,15}=1873.86, p<0.001, \eta_{\mathrm{p}}^{2}=0.992\right)$. These results indicate that our awareness manipulation was effective for both the visible and invisible conditions, and there was no significant difference in subject performance among five distances. Error bars denote 1 SEM calculated across subjects and open dots denote the data from each subject. (C) To manipulate the cueing effect between visible and invisible conditions, we decreased the luminance of the cue in visible condition. Fourteen of our 16 subjects repeated the same 2 AFC test and the results showed that subjects' performances were not statistically different from chance in the invisible condition [Experiment 1 (i.e., varied cue), D0: $48.661 \pm 1.121 \%$, D1: $50.893 \pm 1.652 \%$, D2: 
$47.991 \pm 1.927 \%, \mathrm{D} 3: 49.330 \pm 2.050 \%, \mathrm{D} 4: 48.214 \pm 1.725 \%$, all $\mathrm{t}_{13}<1.194, p>$ $0.254, \eta_{\mathrm{p}}^{2}<0.662$; Experiment 2 (i.e., constant cue): $50.536 \pm 0.536 \%, \mathrm{t}_{13}=1.000, p=$ $\left.0.336, \eta_{\mathrm{p}}^{2}=0.555\right]$, but were significantly higher than chance in the visible condition (Experiment 1, D0: $85.268 \pm 3.315 \%, \mathrm{D} 1: 83.929 \pm 3.747 \%, \mathrm{D} 2: 77.232 \pm 2.749 \%$, D3: $79.911 \pm 3.774 \%, \mathrm{D} 4: 78.571 \pm 2.336 \%$, all $\mathrm{t}_{13}>7.926, p<0.001, \eta_{\mathrm{p}}^{2}>4.397$; Experiment 2: $\left.73.036 \pm 1.086 \%, \mathrm{t}_{13}=21.207, p<0.001, \eta_{\mathrm{p}}^{2}=11.764\right)$. Similarly, for the Experiment 1, subjects' performances were also submitted to a repeated-measures ANOVA with awareness (visible and invisible) and distance (D0 to D4) as within-subjects factors. The main effect of distance $\left(\mathrm{F}_{4,52}=1.892, p=0.126, \eta_{\mathrm{p}}^{2}=\right.$ $0.127)$ and the interaction between the two factors $\left(\mathrm{F}_{4,52}=0.923, p=0.458, \eta_{\mathrm{p}}^{2}=\right.$ 0.066) were not significant, but the main effect of awareness was significant $\left(\mathrm{F}_{1,13}=\right.$ 171.254, $\left.p<0.001, \eta_{\mathrm{p}}^{2}=0.929\right)$. These results further conform that our awareness manipulation was effective for both the visible and invisible conditions, and there was no significant difference in subject performance among five distances. Error bars denote 1 SEM calculated across subjects and open dots denote the data from each subject. 
A Visible Invisible B
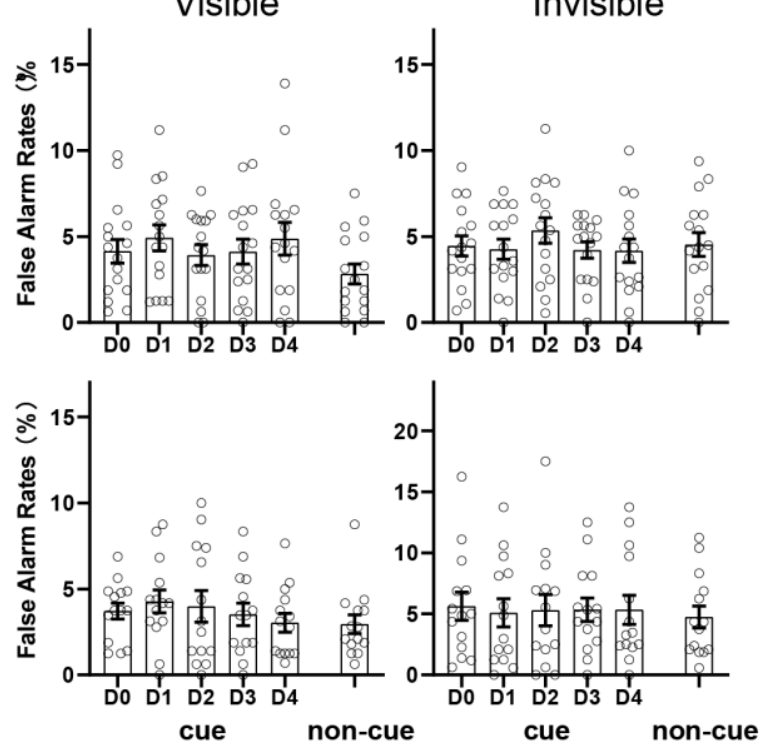

C
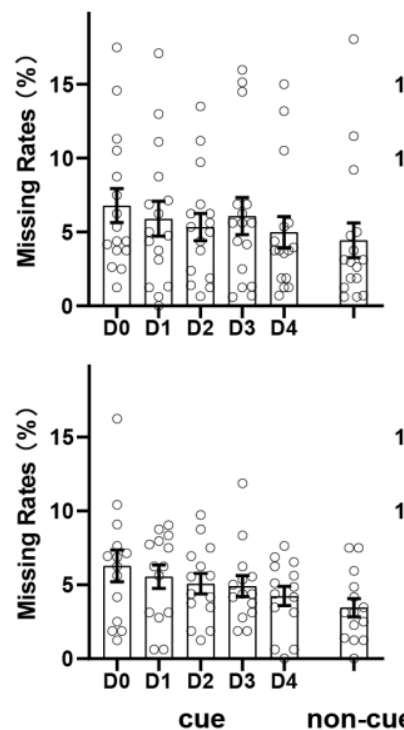

$\mathrm{E}$
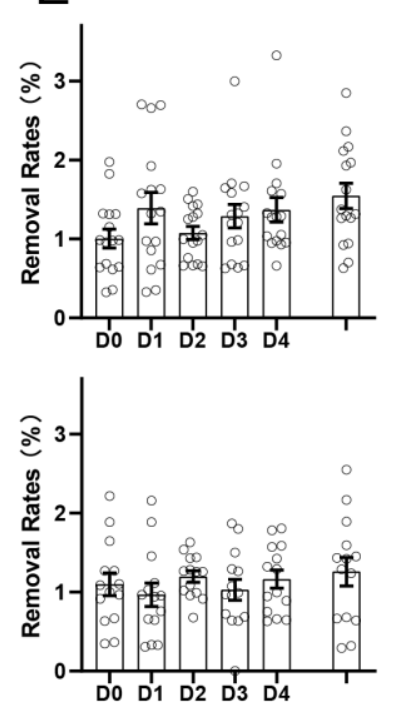

cue
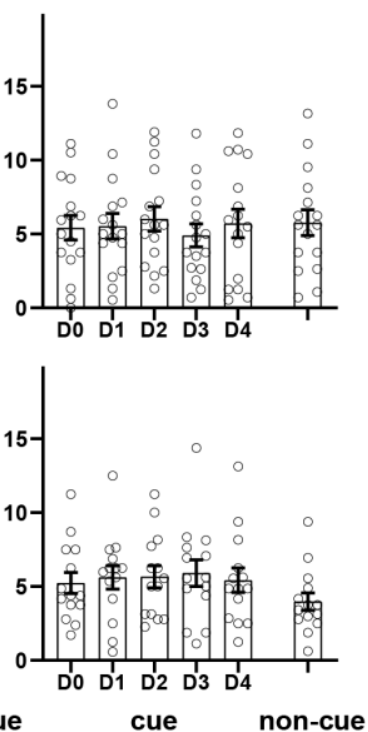

cue non-cue
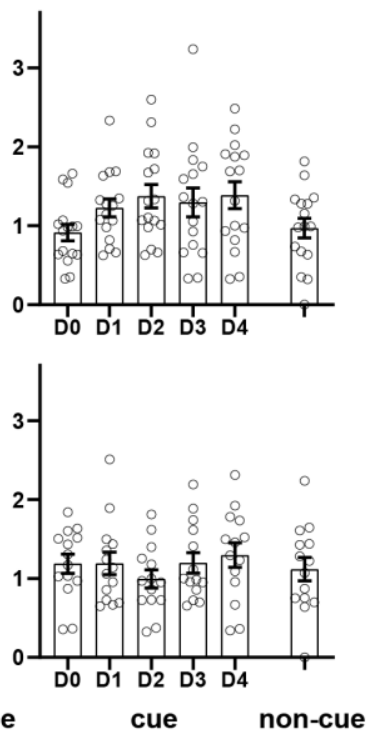

D

$\mathrm{F}$
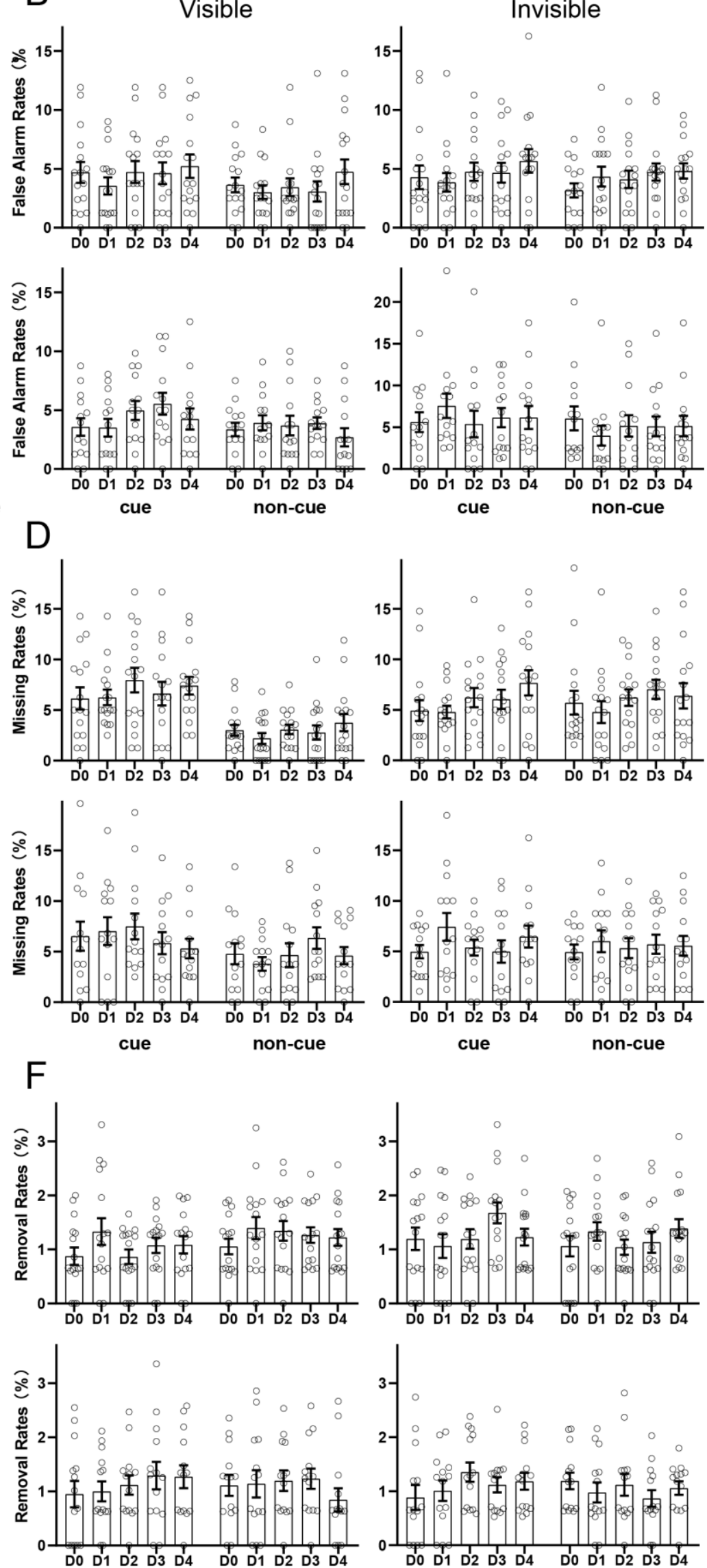

cue

non-cue
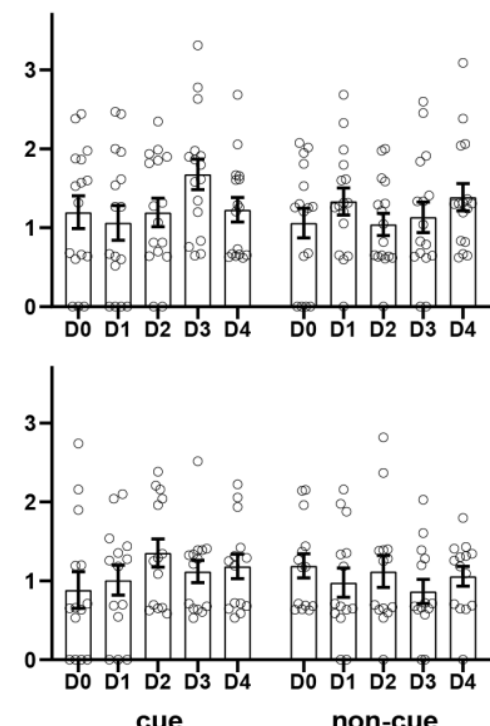
Figure S2. False alarm, Miss, and Removal Rates in Distribution Experiments. False alarm rates of the cue and non-cue conditions for each distance (D0 to D4) during visible (left) and invisible (right) conditions in Experiments 1 (A) and 2 (B). Note that, in our study, subjects were asked to press one of two buttons as rapidly and correctly as possible to indicate the orientation of the line probe $\left(45^{\circ}\right.$ or $\left.135^{\circ}\right)$. Thus, for each condition, a rightward response to a $45^{\circ}$ line was (arbitrarily) considered to be a hit, a rightward response to a $135^{\circ}$ line was considered to be a false alarm, and a leftward response to a $45^{\circ}$ line was considered to be a miss. Error bars denote 1 SEM calculated across subjects and open dots denote the data from each subject. Miss rates of the cue and non-cue conditions for each distance (D0 to D4) during visible (left) and invisible (right) conditions in Experiments 1 (C) and 2 (D). Error bars denote 1 SEM calculated across subjects and open dots denote the data from each subject. Removal rates (i.e., correct reaction times shorter than $200 \mathrm{~ms}$ and beyond three standard deviations from the mean reaction time in each condition were removed) of the cue and non-cue conditions for each distance (D0 to D4) during visible (left) and invisible (right) conditions in Experiments 1 (E) and 2 (F). Error bars denote 1 SEM calculated across subjects and open dots denote the data from each subject. There was no significant difference in false alarm rate, miss rate, or removal rate across conditions (all $P>0.05)$. 
A

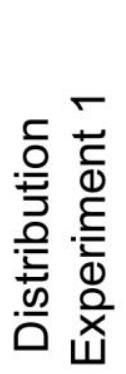

Visible
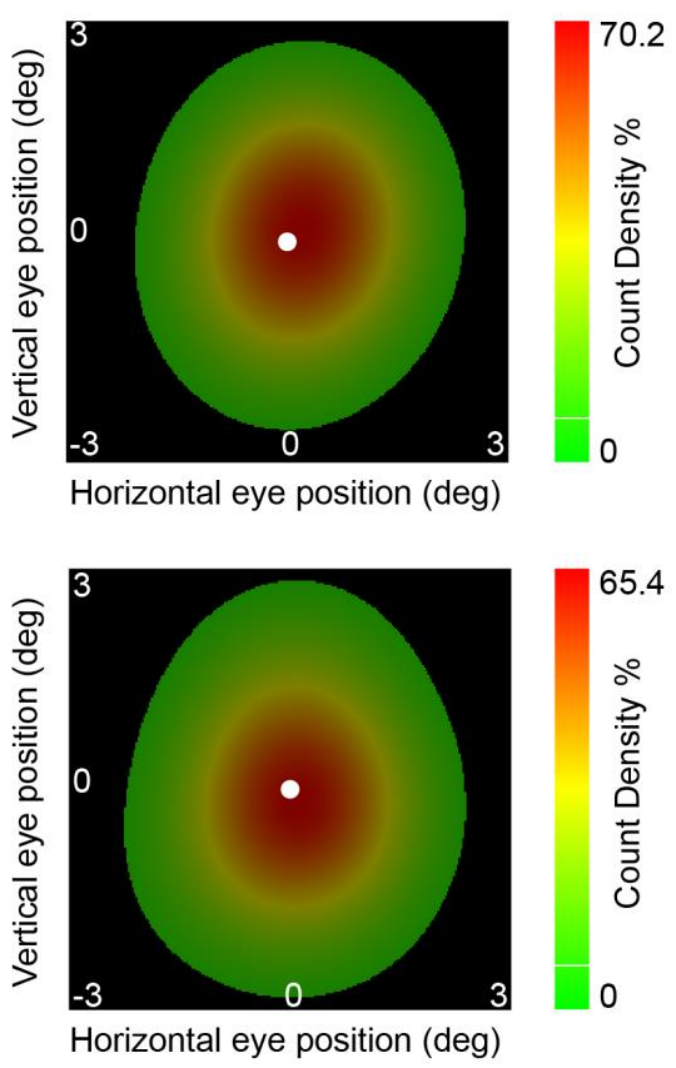

B

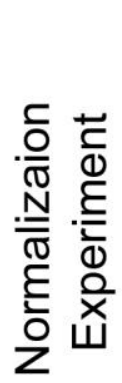

Visible

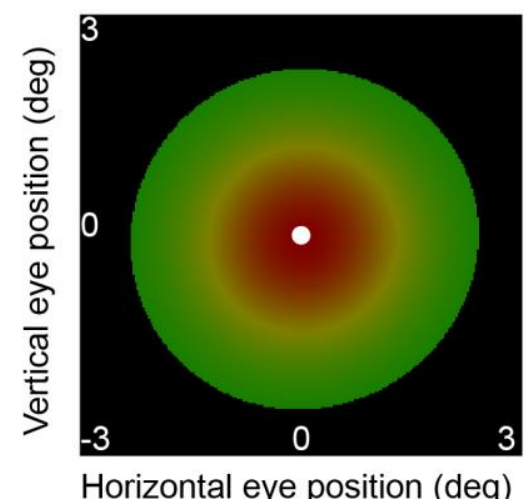

Invisible
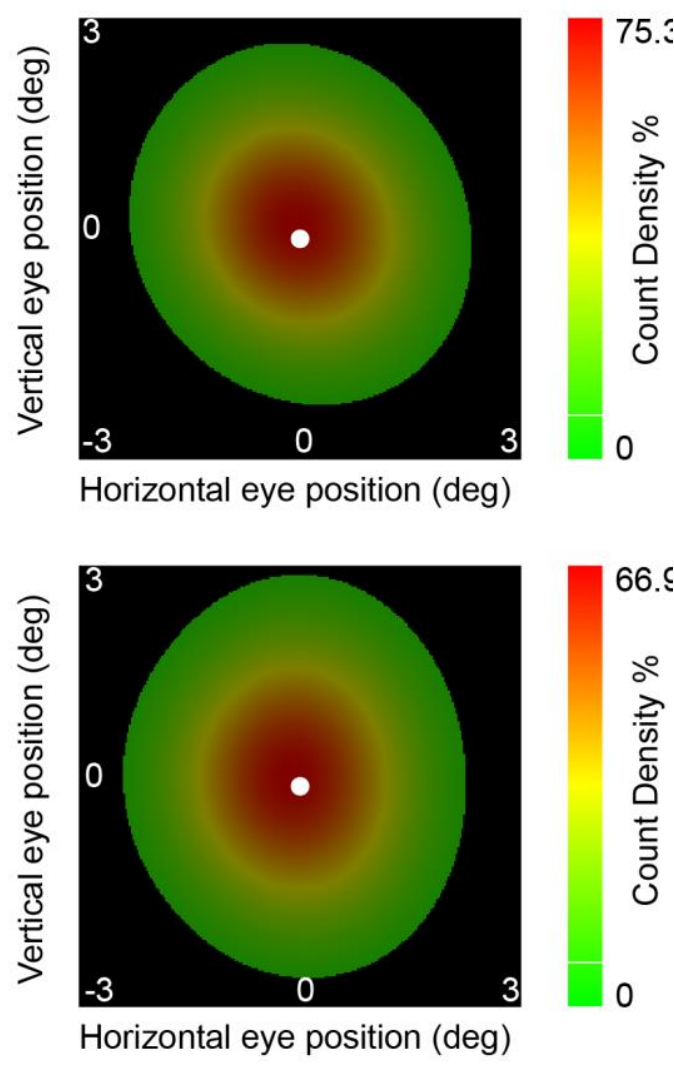

Invisible

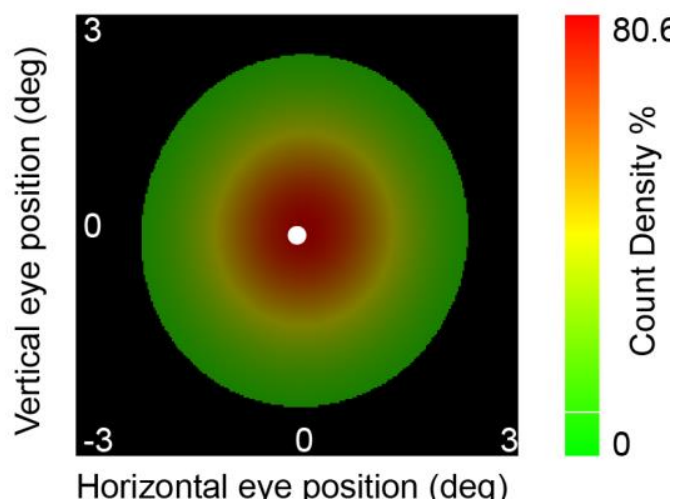

Figure S3. Eye Movement Data in Distribution and Normalization Experiments.

Horizontal and vertical eye positions after removing blinks and artifacts of the visible

(left) and invisible (right) conditions in Distribution (A) and Normalization (B)

Experiments. Subjects` eye movements were small $\left(<3^{\circ}\right)$ and not systematically different between the visible and invisible conditions (all $p>0.05$ ). 

Visible
Invisible
Visible
Invisible

A
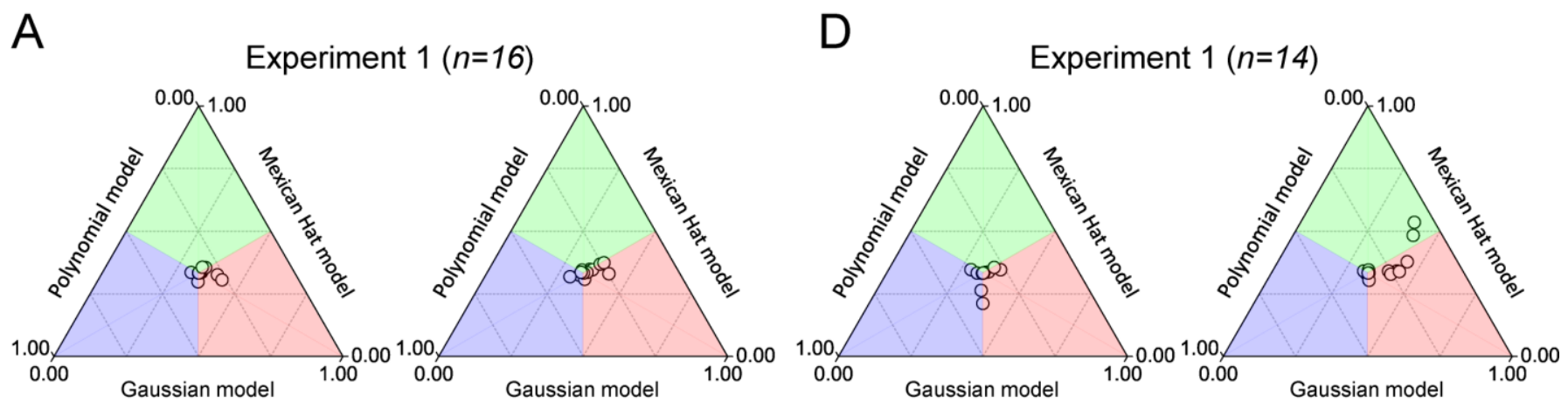

B

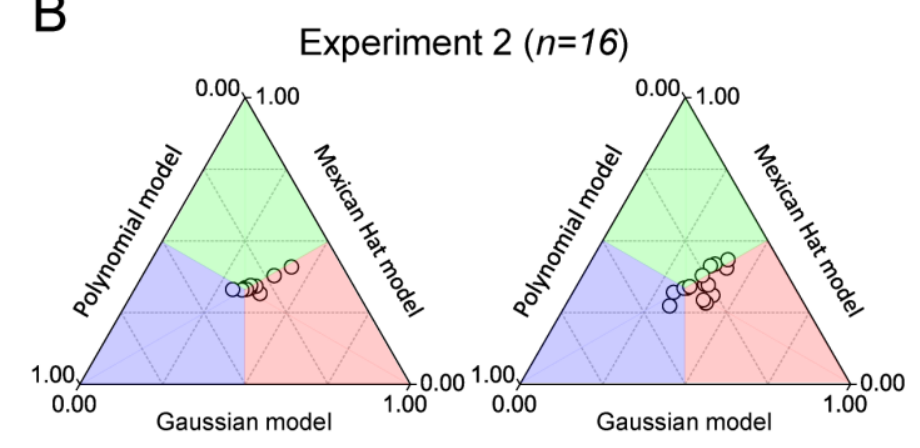

E Experiment $2(n=14)$

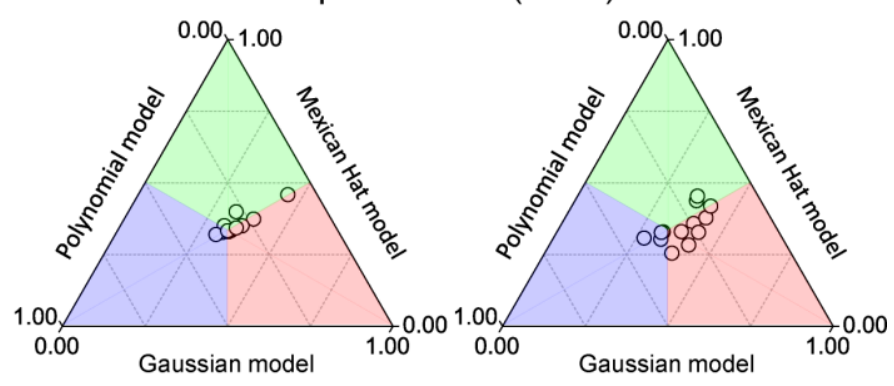

C
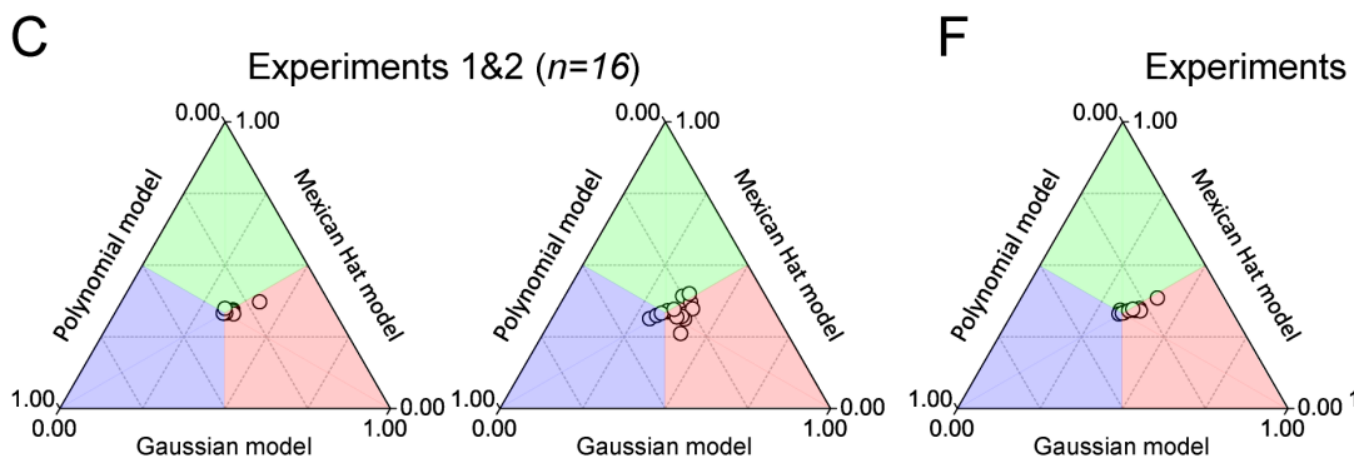

Experiments $1 \& 2(n=14)$

Figure S4. Normalized $\mathrm{R}^{2}$ of Individual Subjects in Distribution Experiments. In

Experiment 1 (A and D), Experiment $2(\mathbf{B}$ and $\mathbf{E})$, and Experiments $1 \& 2(\mathbf{C}$ and $\mathbf{F})$,

to directly compare the fitted $\mathrm{R}^{2}$ among Gaussian, Mexican Hat, and Polynomial models, we normalized the $\mathrm{R}^{2}$ of each subject to $R_{G}^{2}+R_{M}^{2}+R_{P}^{2}=1$, where $R_{G}^{2}, R_{M}^{2}$, and $R_{P}^{2}$ was the fitted $\mathrm{R}^{2}$ using the Gaussian, Mexican Hat, and Polynomial functions, respectively. During each condition (left: visible; right: invisible) and each experiment, most of the dots are located in the red zone, demonstrating that the Gaussian model was favored over both the Mexican Hat and Polynomial models. 
A

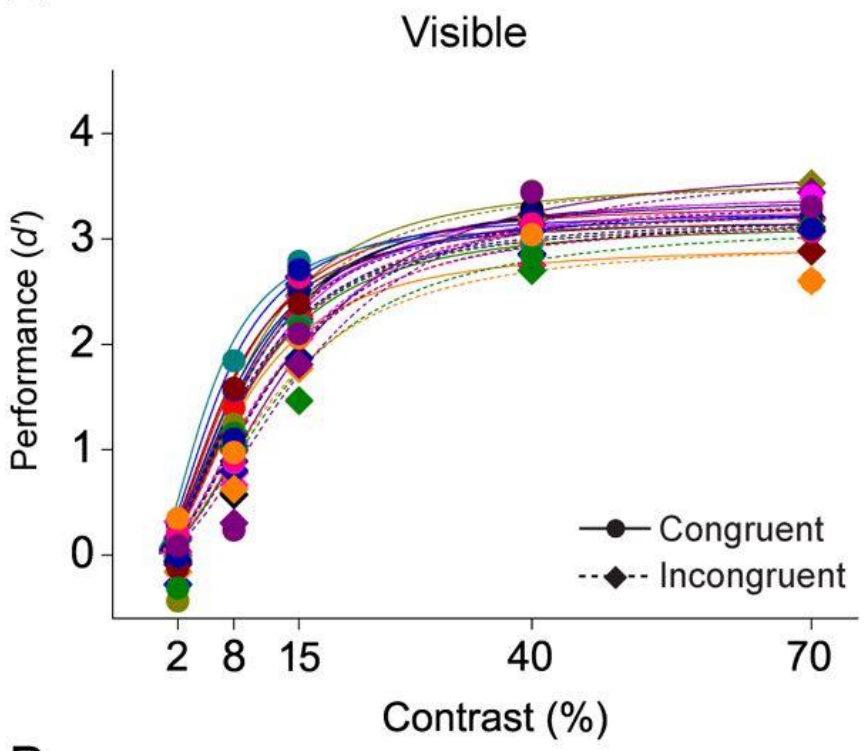

B

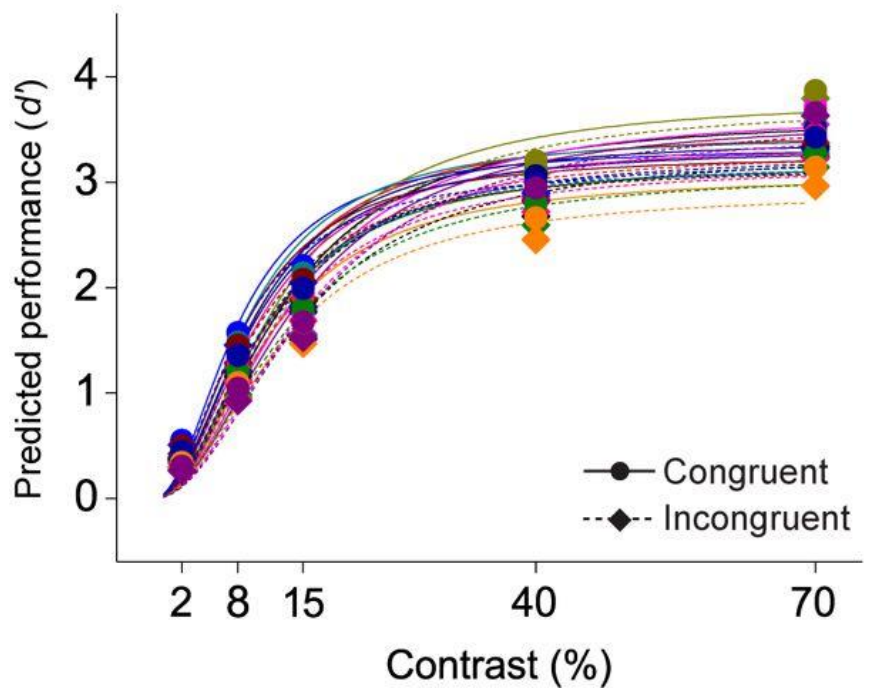

Invisible
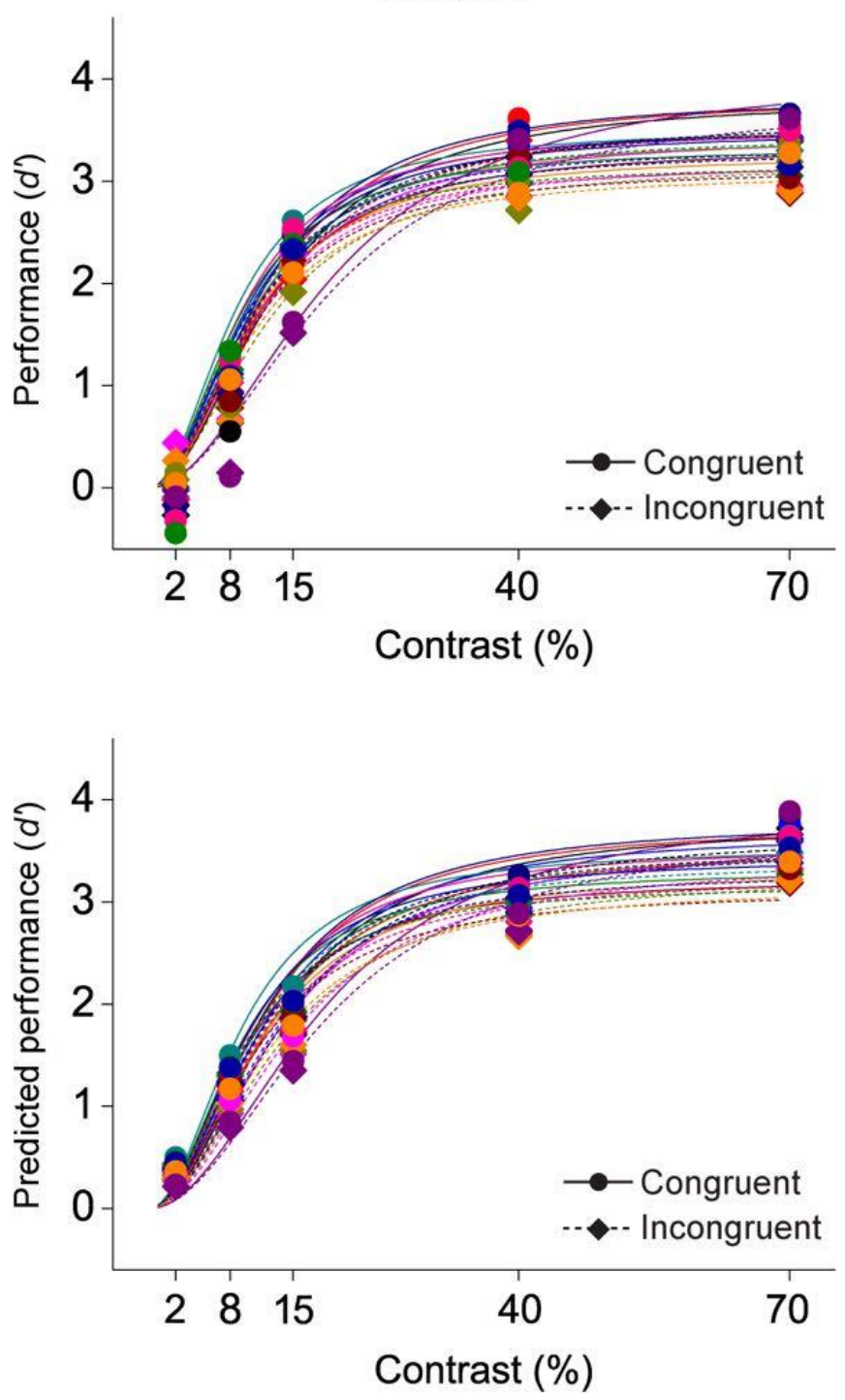

Figure S5. Contrast Response Functions of Individual Subjects in Normalization Experiments. Data points are experimental (A) and predicted (B) performances $\left(d^{\prime}\right)$ of individual subjects for awareness (visible and invisible), cue validity (congruent and incongruent), and stimulus contrasts. The individual contrast response function was fitted using the standard Naka-Rushton equation (A) and the original normalization model of attention with 4 free parameters (B), respectively. 